

\title{
LEGENDRE SPECTRAL-COLLOCATION METHOD FOR VOLTERRA INTEGRAL DIFFERENTIAL EQUATIONS WITH NONVANISHING DELAY
}

\author{
YANPING CHEN AND ZHENDONG GU
}

\begin{abstract}
The main purpose of this paper is to propose the Legendre spectral-collocation method to solve the Volterra integral differential equations with nonvanishing delay which arise in many problems, such as modeling in biosciences and population. In our method we divide the definition domain of the solution into several subintervals where the solution is sufficiently smooth. Then we can use the spectral-collocation method for these equations in each subinterval. We provide convergence analysis for this method, which shows that the numerical errors decay exponentially. Numerical examples are presented to confirm these theoretical results.
\end{abstract}

\section{Introduction}

VIDEs (Volterra integral differential equations) with delay arise in many problems, for example, ecological competition systems [54], modeling in biosciences and population $[1 ; 9 ; 26]$ and models for transmission of disease with immigration of infectives [10]. Nonlinear Volterra integral and integrodifferential equations with nonvanishing delay have been used since the 1920s as mathematical models of population growth and related phenomena in biology. Volterra [46] refined his earlier predator-prey model to include situations where "historical actions cease after a certain interval of time" [47]. This leads to a system of nonlinear Volterra

Yanping Chen is the corresponding author.

This work is supported by National Science Foundation of China (11271145), Foundation for Talent Introduction of Guangdong Provincial University, Specialized Research Fund for the Doctoral Program of Higher Education (20114407110009), the Project of Department of Education of Guangdong Province (2012KJCX0036), Hunan Provincial Innovation Foundation for Postgraduate (CX2012B241).

PACS: primary 65M70; secondary 45D05, $45 \mathrm{~J} 05$.

Keywords: Volterra integral differential equations, nonvanishing delay, Legendre spectral-collocation method, convergence analysis. 
integrodifferential equations with constant delay $T_{0}>0$ (using Volterra's notation):

$$
\begin{aligned}
& N_{1}^{\prime}(t)=N_{1}(t)\left(\varepsilon_{1}-\gamma_{1} N_{2}(t)-\int_{t-T_{0}}^{t} F_{1}(t-\tau) N_{1}(\tau) d \tau\right), \\
& N_{2}^{\prime}(t)=N_{2}(t)\left(-\varepsilon_{2}+\gamma_{2} N_{1}(t)+\int_{t-T_{0}}^{t} F_{2}(t-\tau) N_{2}(\tau) d \tau\right),
\end{aligned}
$$

with $\varepsilon_{i}>0, \gamma_{i} \geq 0$ and continuous $F_{i}(t) \geq 0$, where $N_{i}(t)=\phi_{i}(t), t \leq 0, i=1,2$. $N_{1}(t)$ and $N_{2}(t)$ represent the sizes of two populations (prey and predator) at time $t \geq 0$. These equations can be extended naturally to describe the dynamics of multispecies ecological systems. A further development of such population models based on VIDEs can be found in [20].

There exist many numerical methods for the VIDEs with delay, for example, general linear methods [53], linear multistep methods [5], block-by-block methods [32], Runge-Kutta methods [6; 7; 21;29; 38], Petrov-Galerkin methods [31], piecewise polynomial collocation methods $[13 ; 14 ; 36 ; 37]$. Brunner investigated the numerical solution of nonlinear VIDEs with infinite delay in [11] and neutral VIDEs with constant delay in [12]. The superconvergence of the collocation method for VIDEs with nonvanishing delay is investigated in $[13 ; 37 ; 39]$. The monograph by Brunner [13] contains a wealth of material on the theory and numerical methods for VIDEs, with the focus being on the basic theory of Volterra equations with delay and the collocation methods and their convergence analysis.

Without the integral terms in VIDEs we obtain DDEs (delay differential equations). DDE models arise in many problems, such as the growth of tumors [45], population dynamics [28], hepatitis B virus infection [23], harmful algal blooms in the presence of toxic substances [16]. More applications of DDEs are described in [28]. Numerically solving DDEs has many of the same difficulties discussed for delay VIDEs. Many numerical methods are investigated for DDEs [6; 22; 34; 55]. The monograph by Bellen and Zennaro [8] gives a comprehensive account of numerical methods for DDEs, with the focus being on (classical and continuous) Runge-Kutta methods and their asymptotic stability properties which were also investigated by Baker and Tang [7]. There are some well-developed softwares for delay differential equations or systems. The popular solver developed by Shampine and Thompson [40; 44] for DDEs is well tested and user-friendly.

Spectral methods receive considerable attention mainly due to their high accuracy. Tang, Xu and Cheng [43] proposed a Legendre spectral-collocation method to solve VIEs (Volterra integral equations) of the second kind whose kernel and solutions are sufficiently smooth. Chen and Tang $[17 ; 18 ; 19]$ proposed and analyzed a Jacobi spectral-collocation approximation for linear VIEs of the second kind with weakly singular kernels provided that the underlying solutions of the VIEs are sufficiently smooth. Then, in [30], the Jacobi spectral-collocation method was extended to 
solve VIEs with Abel-type kernel. Recently, another spectral method, i.e., the Legendre spectral Galerkin method, was investigated in [48; 52] for VIEs. The spectral-collocation methods also attract the interest of those people who study the Volterra-type integral and related functional differential equations (see, e.g., [2; 3; $4 ; 27 ; 42 ; 49 ; 50 ; 51])$.

However, there is very little literature about the spectral method to solve VIDEs with nonvanishing delay. The main difficulty in applying the spectral method to VIDEs with nonvanishing delay is that the solutions of these equations are not smooth enough at the primary discontinuous points associated with the delay function. In this paper, we overcome this difficulty and propose a Legendre spectralcollocation method to solve these equations. In our method we divide the definition domain into several subintervals according to the primary discontinuous points associated with the nonvanishing delay function. In each subinterval, where the solution is smooth enough, we can apply the Legendre spectral-collocation method to approximate the solution. We provide convergence analysis to show that the numerical errors decay exponentially. Numerical examples are presented to confirm this theoretical prediction.

The VIDEs with nonvanishing delay considered in this paper are as follows:

$$
\begin{gathered}
y^{\prime}(t)=a(t) y(t)+b(t) y(\theta(t))+g(t)+\int_{0}^{t} K_{1}(t, s) y(s) d s+\int_{0}^{\theta(t)} K_{2}(t, s) y(s) d s, \\
t \in(0, T], \\
y(t)=\phi(t), \quad t \in[\theta(0), 0] .
\end{gathered}
$$

In population models, $y(t)$ means the population size at time $t$. The delay $\theta(t)$ means that the growth of population size depends on the historical action. We assume that the functions describing the above equation all possess continuous derivatives of at least order $m \geq 1$ on their respective domains; i.e.,

$$
\begin{gathered}
a(t), b(t), g(t) \in C^{m}([0, T]), \quad \phi(t) \in C^{m}([\theta(0), 0]), \\
K_{1}(t, s) \in C^{m}\left(\Omega_{1}\right), \quad \Omega_{1}:=\{(t, s): 0 \leq s \leq t \leq T\}, \\
K_{2}(t, s) \in C^{m}\left(\Omega_{2}\right), \quad \Omega_{2}:=\{(t, s): \theta(0) \leq s \leq \theta(t), 0 \leq t \leq T\},
\end{gathered}
$$

and the delay function $\theta$ will be subject to the following conditions:

$$
\begin{gathered}
\theta(t):=t-\tau(t), \quad \tau \in C^{m}([0, T]), \\
\tau(t) \geq \tau_{0}>0 \text { for all } t \in[0, T], \\
\theta \text { is strictly increasing on }[0, T] .
\end{gathered}
$$

The nonvanishing delay $\theta$ gives rise to the primary discontinuity points $\left\{\xi_{\mu}\right\}$ for the solution of (1): they are determined by the recursion

$$
\theta\left(\xi_{\mu}\right)=\xi_{\mu-1}, \quad \mu \geq 0 \quad\left(\xi_{-1}:=\theta(0), \xi_{0}=0\right) .
$$


These points have the uniform separation property

$$
\xi_{\mu}-\xi_{\mu-1} \geq \tau_{0}>0 \text { for all } \mu \geq 0 .
$$

For ease of notation we will assume that

$$
T=\xi_{M+1} \quad \text { for some } M \geq 1 .
$$

Theorem 4.1.9 in [13] states that the unique solution of (1) is in $C^{m+1}\left(\xi_{\mu}, \xi_{\mu+1}\right.$ ] for each $\mu=0,1, \ldots, M$ and is bounded on $Z_{M}:=\left\{\xi_{\mu}: \mu=0,1, \ldots, M\right\}$ and hence on $[0, T]$. At $t=\xi_{\mu}(\mu=1, \ldots, \min \{m, M\})$,

$$
\lim _{t \rightarrow \xi_{\mu}^{-}} y^{(\mu)}(t)=\lim _{t \rightarrow \xi_{\mu}^{+}} y^{(\mu)}(t),
$$

while the $(\mu+1)$-th derivative of $y$ is in general not continuous at $\xi_{\mu}$. In addition, if $\min \{m, M\}=m<M$, the solution also lies in $C^{m+1}\left[\xi_{m}, T\right]$. This motivates us to apply the spectral-collocation method to approximate the solution on the subinterval $\left(\xi_{\mu}, \xi_{\mu+1}\right], \mu=0,1, \ldots, M$.

This paper is organized as follows. In Section 2, we introduce the Legendre spectral-collocation method for VIDEs with nonvanishing delay. Some useful lemmas for the convergence analysis will be provided in Section 4, and the convergence analysis, in both $L^{\infty}$ and $L^{2}$, will be given in Section 5. Numerical experiments are carried out in Section 6. Finally, in Section 7, we end with the conclusion and future work.

\section{Legendre spectral-collocation method}

For ease of analysis we change the interval $[0, T]$ to the standard interval $[-1,1]$. Precisely we use the variable transformation

$$
t(x)=\frac{1}{2} T(x+1), \quad s(z)=\frac{1}{2} T(z+1) .
$$

Then (1) can be written as

$$
\begin{aligned}
& u^{\prime}(x)=A(x) u(x)+B(x) u(\vartheta(x))+f(x) \\
& \quad+\int_{-1}^{x} R_{1}(x, z) u(z) d z+\int_{-1}^{\vartheta(x)} R_{2}(x, z) u(z) d z, \quad x \in(-1,1], \\
& u(x)=\psi(x), \quad x \in[\vartheta(-1),-1],
\end{aligned}
$$

where

$$
\begin{gathered}
u(x):=y(t(x)), \quad A(x):=\frac{1}{2} T a(t(x)), \quad B(x):=\frac{1}{2} T b(t(x)), \\
f(x):=\frac{1}{2} T g(t(x)), \quad \vartheta(x):=\frac{2}{T} \theta(t(x))-1, \quad \psi(x):=\phi(t(x)), \\
R_{1}(x, z):=\left(\frac{1}{2} T\right)^{2} K_{1}(t(x), s(z)), \quad R_{2}(x, z):=\left(\frac{1}{2} T\right)^{2} K_{2}(t(x), s(z)) .
\end{gathered}
$$


The primary discontinuity point $\xi_{\mu}$ becomes

$$
\eta_{\mu}:=\left(2 \xi_{\mu} / T\right)-1, \quad \mu=-1,0,1, \ldots, M .
$$

Define

$$
\delta_{\mu}:=\left(\eta_{\mu}, \eta_{\mu+1}\right], \quad \mu=-1,0, \ldots, M .
$$

Set the collocation points as follows:

$$
X_{N}:=\bigcup_{\mu=0}^{M} X^{\mu}, \quad X^{\mu}:=\left\{x_{n}^{\mu}: \eta_{\mu}=x_{0}^{\mu}<x_{1}^{\mu}<\cdots<x_{N}^{\mu}=\eta_{\mu+1}\right\},
$$

where

$$
x_{i}^{\mu}:=\frac{\eta_{\mu+1}-\eta_{\mu}}{2} x_{i}+\frac{\eta_{\mu+1}+\eta_{\mu}}{2} ;
$$

here $x_{i}, i=0,1, \ldots, N$, are the $N+1$ Legendre Gauss-Lobatto points in the standard interval $[-1,1]$. Then (5) holds at $x_{i}^{\mu}, i=0,1, \ldots, N, \mu=0,1, \ldots, M$ :

$$
\begin{aligned}
u^{\prime}\left(x_{i}^{\mu}\right)=A\left(x_{i}^{\mu}\right) u\left(x_{i}^{\mu}\right)+ & B\left(x_{i}^{\mu}\right) u\left(\vartheta\left(x_{i}^{\mu}\right)\right)+f\left(x_{i}^{\mu}\right) \\
& +\int_{-1}^{x_{i}^{\mu}} R_{1}\left(x_{i}^{\mu}, z\right) u(z) d z+\int_{-1}^{\vartheta\left(x_{i}^{\mu}\right)} R_{2}\left(x_{i}^{\mu}, z\right) u(z) d z .
\end{aligned}
$$

We use $u_{i}^{\mu}$ to approximate $u\left(x_{i}^{\mu}\right), v_{i}^{\mu}$ to approximate $u\left(\vartheta\left(x_{i}^{\mu}\right)\right), \rho_{i}^{\mu}$ to approximate $u^{\prime}\left(x_{i}^{\mu}\right)$. Then we can use

$$
u_{\mu}(x):=\sum_{j=0}^{N} u_{j}^{\mu} F_{j}^{\mu}(x), \quad x \in\left[\eta_{\mu}, \eta_{\mu+1}\right]
$$

to approximate $\left.u\right|_{\delta_{\mu}}(x)$, i.e., the restriction of $u(x)$ to the interval $\left[\eta_{\mu}, \eta_{\mu+1}\right]$. $F_{j}{ }^{\mu}(x), x \in\left[\eta_{\mu}, \eta_{\mu+1}\right]$, is the $j$-th Lagrange interpolation basic function associated with the collocation points $x_{0}^{\mu}, x_{1}^{\mu}, \ldots, x_{N}^{\mu}$ in the interval $\left[\eta_{\mu}, \eta_{\mu+1}\right]$. Similarly, we use

$$
\rho_{\mu}(x):=\sum_{j=0}^{N} \rho_{j}^{\mu} F_{j}^{\mu}(x), \quad x \in\left[\eta_{\mu}, \eta_{\mu+1}\right]
$$

to approximate $\left.u^{\prime}\right|_{\delta_{\mu}}(x)$, i.e., the restriction of $u^{\prime}(x)$ to the subinterval $\left[\eta_{\mu}, \eta_{\mu+1}\right]$. Eventually $u(x)$ can be approximated by

$$
u^{N}(x):=u_{\mu}(x) \quad \text { if } x \in\left[\eta_{\mu}, \eta_{\mu+1}\right], \mu=0,1, \ldots, M,
$$

and $u^{\prime}(x)$ can be approximated by

$$
\rho^{N}(x):=\rho_{\mu}(x) \quad \text { if } x \in\left[\eta_{\mu}, \eta_{\mu+1}\right], \mu=0,1, \ldots, M .
$$


Then (9) can be approximated by

$$
\begin{aligned}
\rho_{i}^{\mu} \approx A\left(x_{i}^{\mu}\right) u_{i}^{\mu}+ & B\left(x_{i}^{\mu}\right) v_{i}^{\mu}+f\left(x_{i}^{\mu}\right) \\
& +\int_{-1}^{x_{i}^{\mu}} R_{1}\left(x_{i}^{\mu}, z\right) u^{N}(z) d z+\int_{-1}^{\vartheta\left(x_{i}^{\mu}\right)} R_{2}\left(x_{i}^{\mu}, z\right) u^{N}(z) d z,
\end{aligned}
$$

which can be written as

$$
\begin{aligned}
\rho_{i}^{\mu} \approx A\left(x_{i}^{\mu}\right) & u_{i}^{\mu}+B\left(x_{i}^{\mu}\right) v_{i}^{\mu}+f\left(x_{i}^{\mu}\right) \\
& +\sum_{r=0}^{\mu-1} \int_{\eta_{r}}^{\eta_{r+1}} R_{1}\left(x_{i}^{\mu}, z\right) u_{r}(z) d z+\int_{\eta_{\mu}}^{x_{i}^{\mu}} R_{1}\left(x_{i}^{\mu}, z\right) u_{\mu}(z) d z \\
& +\sum_{r=0}^{\mu-2} \int_{\eta_{r}}^{\eta_{r+1}} R_{2}\left(x_{i}^{\mu}, z\right) u_{r}(z) d z+\int_{\eta_{\mu-1}}^{\vartheta\left(x_{i}^{\mu}\right)} R_{2}\left(x_{i}^{\mu}, z\right) u_{\mu-1}(z) d z .
\end{aligned}
$$

In order to compute the integral term by the Gauss quadrature rule, we change the integration interval to the standard interval $[-1,1]$. Note that the variable transformation

$$
z(a, b, v):=\frac{b-a}{2} v+\frac{b+a}{2}, \quad v \in[-1,1]
$$

can change the interval $[a, b]$ to $[-1,1]$. For simplicity, we define

$$
z_{r}(v):=z\left(\eta_{r}, \eta_{r+1}, v\right), \quad v \in[-1,1], r \geq 0 .
$$

Using the Gauss quadrature formula to approximate the integration term in (11) we obtain

$$
\begin{aligned}
\rho_{i}^{\mu}= & A\left(x_{i}^{\mu}\right) u_{i}^{\mu}+B\left(x_{i}^{\mu}\right) v_{i}^{\mu}+f\left(x_{i}^{\mu}\right)+\sum_{r=0}^{\mu-1} \frac{\eta_{r+1}-\eta_{r}}{2} \sum_{k=0}^{N} R_{1}\left(x_{i}^{\mu}, z_{r}\left(v_{k}\right)\right) u_{r}\left(z_{r}\left(v_{k}\right)\right) \omega_{k} \\
& +\frac{\eta_{\mu+1}-\eta_{\mu}}{2} \frac{x_{i}+1}{2} \sum_{k=0}^{N} R_{1}\left(x_{i}^{\mu}, z_{\mu}\left(z\left(-1, x_{i}, v_{k}\right)\right)\right) u_{\mu}\left(z_{\mu}\left(z\left(-1, x_{i}, v_{k}\right)\right)\right) \omega_{k} \\
& +\sum_{r=0}^{\mu-2} \frac{\eta_{r+1}-\eta_{r}}{2} \sum_{k=0}^{N} R_{2}\left(x_{i}^{\mu}, z_{r}\left(v_{k}\right)\right) u_{r}\left(z_{r}\left(v_{k}\right)\right) \omega_{k} \\
& +\frac{\eta_{\mu}-\eta_{\mu-1}}{2} \frac{\widetilde{\vartheta}\left(x_{i}^{\mu}\right)+1}{2} \sum_{k=0}^{N} R_{2}\left(x_{i}^{\mu}, z_{\mu-1}\left(z\left(-1, \widetilde{\vartheta}\left(x_{i}^{\mu}\right), v_{k}\right)\right)\right) \\
& \times u_{\mu-1}\left(z_{\mu-1}\left(z\left(-1, \widetilde{\vartheta}\left(x_{i}^{\mu}\right), v_{k}\right)\right)\right) \omega_{k}
\end{aligned}
$$

where $v_{k}, k=0,1, \ldots, N$, are the $N+1$ Legendre Gauss-Lobatto points in the standard interval $[-1,1]$, corresponding to the weights $\omega_{k}, k=0,1, \ldots, N$, and 


$$
\widetilde{\vartheta}\left(x_{i}^{\mu}\right):=\frac{2}{\eta_{\mu}-\eta_{\mu-1}} \vartheta\left(x_{i}^{\mu}\right)-\frac{\eta_{\mu}+\eta_{\mu-1}}{\eta_{\mu}-\eta_{\mu-1}}, \quad \mu>0 .
$$

Note that, for $j, k=0,1, \ldots, N, r=0,1, \ldots, M$,

$$
\begin{gathered}
F_{j}^{r}\left(z_{r}\left(v_{k}\right)\right)=F_{j}\left(v_{k}\right)= \begin{cases}1, & k=j, \\
0, & k \neq j,\end{cases} \\
F_{j}^{r}\left(z_{r}(z(-1, x, v))\right)=F_{j}(z(-1, x, v)),
\end{gathered}
$$

where $F_{j}(v)$ is the $j$-th Lagrange interpolation basic function associated with the $N+1$ Legendre Gauss-Lobatto points in the standard interval $[-1,1]$. Then (14) can be simplified to

$$
\begin{aligned}
\rho_{i}^{\mu}=A\left(x_{i}^{\mu}\right) u_{i}^{\mu}+B\left(x_{i}^{\mu}\right) v_{i}^{\mu}+f\left(x_{i}^{\mu}\right)+\beta\left(x_{i}^{\mu}\right)+\lambda\left(x_{i}^{\mu}\right), & \\
& \mu=0,1, \ldots, M, i=0,1, \ldots, N,
\end{aligned}
$$

where

$$
\begin{aligned}
\beta\left(x_{i}^{\mu}\right) & :=\sum_{r=0}^{\mu-1} \frac{\eta_{r+1}-\eta_{r}}{2} \beta_{1}^{r}\left(x_{i}^{\mu}\right)+\frac{\eta_{\mu+1}-\eta_{\mu}}{2} \frac{x_{i}+1}{2} \beta_{3}\left(x_{i}^{\mu}\right), \\
\beta_{1}^{r}\left(x_{i}^{\mu}\right) & :=\sum_{k=0}^{N} R_{1}\left(x_{i}^{\mu}, z_{r}\left(v_{k}\right)\right) u_{k}^{r} \omega_{k}, \quad r=0,1, \ldots, \mu-1, \\
\beta_{3}\left(x_{i}^{\mu}\right) & :=\sum_{j=0}^{N} u_{j}^{\mu} \sum_{k=0}^{N} R_{1}\left(x_{i}^{\mu}, z_{\mu}\left(z\left(-1, x_{i}, v_{k}\right)\right)\right) F_{j}\left(z\left(-1, x_{i}, v_{k}\right)\right) \omega_{k},
\end{aligned}
$$

and

$$
\lambda\left(x_{i}^{\mu}\right):=\left\{\begin{array}{cc}
\frac{\vartheta\left(x_{i}^{0}\right)+1}{2} \lambda_{2}\left(x_{i}^{0}\right), & \mu=0, \\
\sum_{r=0}^{\mu-2} \frac{\eta_{r+1}-\eta_{r}}{2} \lambda_{1}^{r}\left(x_{i}^{\mu}\right)+\frac{\eta_{\mu}-\eta_{\mu-1}}{2} \frac{\widetilde{\vartheta}\left(x_{i}^{\mu}\right)+1}{2} \lambda_{3}\left(x_{i}^{\mu}\right), & \mu>0,
\end{array}\right.
$$$$
\lambda_{1}^{r}\left(x_{i}^{\mu}\right):=\sum_{j=0}^{N} u_{j}^{r} R_{2}\left(x_{i}^{\mu}, z_{r}\left(v_{j}\right)\right) \omega_{j}, \quad r=0,1, \ldots, \mu-2 \text {, }
$$$$
\lambda_{2}\left(x_{i}^{0}\right):=\sum_{k=0}^{N} R_{2}\left(x_{i}^{0}, z\left(-1, \vartheta\left(x_{i}^{0}\right), v_{k}\right)\right) \psi\left(z\left(-1, \vartheta\left(x_{i}^{0}\right), v_{k}\right)\right) \omega_{k},
$$$$
\lambda_{3}\left(x_{i}^{\mu}\right):=\sum_{j=0}^{N} u_{j}^{\mu-1} \sum_{k=0}^{N} R_{2}\left(x_{i}^{\mu}, z_{\mu-1}\left(z\left(-1, \widetilde{\vartheta}\left(x_{i}^{\mu}\right), v_{k}\right)\right)\right) F_{j}\left(z\left(-1, \widetilde{\vartheta}\left(x_{i}^{\mu}\right), v_{k}\right)\right) \omega_{k} \text {. }
$$

However, the linear systems (17) alone are not enough to find out the unknown elements. We need two other linear systems associated with $u_{i}^{\mu}, v_{i}^{\mu}, \rho_{i}^{\mu}$, 
$i=0,1, \ldots, N, \mu=0,1, \ldots, M$. Note that

$$
\begin{aligned}
u\left(x_{i}^{\mu}\right)= & u(-1)+\int_{-1}^{x_{i}^{\mu}} u^{\prime}(z) d z \\
= & \psi(-1)+\sum_{r=0}^{\mu-1} \frac{\eta_{r+1}-\eta_{r}}{2} \int_{-1}^{1} u^{\prime}\left(z_{r}(v)\right) d v \\
& \quad+\frac{\eta_{\mu+1}-\eta_{\mu}}{2} \frac{x_{i}+1}{2} \int_{-1}^{1} u^{\prime}\left(z_{\mu}\left(z\left(-1, x_{i}, v\right)\right)\right) d v .
\end{aligned}
$$

Then we can approximate the above equation by

$$
u_{i}^{\mu}=\psi(-1)+\alpha_{1}\left(x_{i}^{\mu}\right), \quad \mu=0,1, \ldots, M, i=0,1, \ldots, N,
$$

where

$$
\begin{aligned}
\alpha_{1}\left(x_{i}^{\mu}\right)=\sum_{r=0}^{\mu-1} \frac{\eta_{r+1}-\eta_{r}}{2} & \sum_{k=0}^{N} \rho_{k}^{r} \omega_{k} \\
& +\frac{\eta_{\mu+1}-\eta_{\mu}}{2} \frac{x_{i}+1}{2} \sum_{j=0}^{N} \rho_{j}^{\mu} \sum_{k=0}^{N} F_{j}\left(z\left(-1, x_{i}, v_{k}\right)\right) \omega_{k} .
\end{aligned}
$$

Similarly, the equation

$$
\begin{aligned}
u\left(\vartheta\left(x_{i}^{\mu}\right)\right)= & u(-1)+\int_{-1}^{\vartheta\left(x_{i}^{\mu}\right)} u^{\prime}(z) d z \\
=\psi(-1)+\sum_{r=0}^{\mu-2} \frac{\eta_{r+1}-\eta_{r}}{2} \int_{-1}^{1} u^{\prime}\left(z_{r}(v)\right) d v & \\
& \quad+\frac{\eta_{\mu}-\eta_{\mu-1}}{2} \frac{\widetilde{\vartheta}\left(x_{i}^{\mu}\right)+1}{2} \int_{-1}^{1} u^{\prime}\left(z_{\mu-1}\left(z\left(-1, \tilde{\vartheta}\left(x_{i}^{\mu}\right), v\right)\right)\right) d v
\end{aligned}
$$

can be approximated by

$$
v_{i}^{\mu}=\psi(-1)+\alpha_{2}\left(x_{i}^{\mu}\right), \quad \mu=0,1, \ldots, M, i=0,1, \ldots, N,
$$

where

$$
\alpha_{2}\left(x_{i}^{\mu}\right)= \begin{cases}\psi\left(\vartheta\left(x_{i}^{0}\right)\right)-\psi(-1), & \mu=0, \\ \sum_{r=0}^{\mu-2} \frac{\eta_{r+1}-\eta_{r}}{2} \sum_{k=0}^{N} \rho_{k}^{r} \omega_{k} & \\ +\frac{\eta_{\mu}-\eta_{\mu-1}}{2} \frac{\widetilde{\vartheta}\left(x_{i}^{\mu}\right)+1}{2} \sum_{j=0}^{N} \rho_{j}^{\mu-1} \sum_{k=0}^{N} F_{j}\left(z\left(-1, \widetilde{\vartheta}\left(x_{i}^{\mu}\right), v_{k}\right)\right) \omega_{k}, & \mu>0 .\end{cases}
$$

Equations (19) and (22) are two linear systems we want to find.

The Legendre spectral-collocation method is to find $\rho_{i}^{\mu}, u_{i}^{\mu}, i=0,1, \ldots, N$, $\mu=0,1,2, \ldots, M$, which satisfy (17), (19) and (22). The approximation to $y(t)$ 
is $u^{N}(2 t / T-1)$; the approximation to $y^{\prime}(t)$ is $(2 / T) \rho^{N}(2 t / T-1)$. An efficient computation of $F_{j}(s)$ can be found in [15] or [43].

\section{The existence of the solution to the discrete system}

In this section we will discuss the existence of the solution to the discrete system (17), (19) and (22). We write the linear system (17), (19) and (22) into matrix form:

$$
\left\{\begin{array}{l}
U^{\prime(\mu)}=\Phi_{1}^{(\mu)}+A^{(\mu)} U^{(\mu)}+R_{1}^{(\mu)} U^{(\mu)}+B^{(\mu)} V^{(\mu)}, \\
U^{(\mu)}=\Phi_{2}^{(\mu)}+\frac{\eta_{\mu+1}-\eta_{\mu}}{2} R_{3}^{(\mu)} U^{\prime \mu}, \\
V^{(\mu)}=\Phi_{3}^{(\mu)}+R_{4}^{(\mu)} U^{\prime \mu-1},
\end{array}\right.
$$

where

$$
\begin{aligned}
U^{\prime(\mu)} & :=\left[\rho_{0}^{\mu}, \rho_{1}^{\mu}, \ldots, \rho_{N}^{\mu}\right]^{\prime}, \\
U^{(\mu)} & :=\left[u_{0}^{\mu}, u_{1}^{\mu}, \ldots, u_{N}^{\mu}\right]^{\prime}, \\
V^{(\mu)} & :=\left[v_{0}^{\mu}, v_{1}^{\mu}, \ldots, v_{N}^{\mu}\right]^{\prime}, \quad \mu>0, \\
V^{(0)} & :=\left[\psi\left(\vartheta\left(x_{0}^{0}\right)\right), \psi\left(\vartheta\left(x_{1}^{0}\right)\right), \ldots, \psi\left(\vartheta\left(x_{N}^{0}\right)\right)\right]^{\prime}, \quad \mu=0, \\
\Phi_{1}^{(\mu)} & :=F^{(\mu)}+\sum_{r=0}^{\mu-1} R_{1}^{(r)} U^{(r)}+\sum_{r=0}^{\mu-2} R_{2}^{(r)} U^{(r)}+R_{2}^{(\mu)} U^{(\mu-1)}, \quad \mu>0, \\
\Phi_{1}^{(0)}(i) & :=F^{(0)}(i)+\frac{\vartheta\left(x_{i}^{0}\right)+1}{2} \sum_{k=0}^{N} R_{2}\left(x_{i}^{0}, z\left(-1, \vartheta\left(x_{i}^{0}\right), v_{k}\right)\right) \\
\Phi_{2}^{(\mu)} & :=\psi(-1)[1,1, \ldots, 1]^{\prime}+\sum_{r=0}^{\mu-1} R_{3}^{(r)} U^{(r)}, \\
\Phi_{3}^{(\mu)} & :=\psi(-1)[1,1, \ldots, 1]^{\prime}+\sum_{r=0}^{\mu-2} R_{3}^{(r)} U^{(r)}, \\
F^{(\mu)} & :=\left[f\left(x_{0}^{\mu}\right), f\left(x_{1}^{\mu}\right), \ldots, f\left(x_{N}^{\mu}\right)\right]^{\prime}, \\
A^{(\mu)} & \left.:=\operatorname{diag}\left[A\left(x_{0}^{\mu}\right), A\left(x_{1}^{\mu}\right), \ldots, v_{k}\right)\right) \omega_{k}, \\
B^{(\mu)} & :=\operatorname{diag}\left[B\left(x_{0}^{\mu}\right), B\left(x_{1}^{\mu}\right), \ldots, B\left(x_{N}^{\mu}\right)\right], \\
R_{j}^{(r)}(i, k) & :=\frac{\eta_{r+1}-\eta_{r}}{2} R_{j}\left(x_{i}^{\mu}, z_{r}\left(v_{k}\right)\right) \omega_{k}, \quad j=1,2, r=0,1, \ldots, \mu-1, \\
R_{3}^{(r)}(i, k) & :=\frac{\eta_{r+1}-\eta_{r}}{2} \omega_{k}, \quad r=0,1, \ldots, \mu-1, \\
R_{1}^{(\mu)}(i, j) & :=\frac{\eta_{\mu+1}-\eta_{\mu} x_{i}+1}{2} \sum_{k=0}^{N} R_{1}\left(x_{i}^{\mu}, z_{\mu}\left(z\left(-1, x_{i}, v_{k}\right)\right)\right) F_{j}\left(z\left(-1, x_{i}, v_{k}\right)\right) \omega_{k},
\end{aligned}
$$


$R_{2}^{(\mu)}(i, j):=\frac{\eta_{\mu}-\eta_{\mu-1}}{2} \frac{\widetilde{\vartheta}\left(x_{i}^{\mu}\right)+1}{2} \sum_{k=0}^{N} R_{2}\left(x_{i}^{\mu}, z_{\mu-1}\left(z\left(-1, \widetilde{\vartheta}\left(x_{i}^{\mu}\right), v_{k}\right)\right)\right)$
$\times F_{j}\left(z\left(-1, \widetilde{\vartheta}\left(x_{i}^{\mu}\right), v_{k}\right)\right) \omega_{k}$, $R_{3}^{(\mu)}(i, j):=\frac{x_{i}+1}{2} \sum_{k=0}^{N} F_{j}\left(z\left(-1, x_{i}, v_{k}\right)\right) \omega_{k}$, $R_{4}^{(\mu)}(i, j):=\frac{\eta_{\mu}-\eta_{\mu-1}}{2} \frac{\widetilde{\vartheta}\left(x_{i}^{\mu}\right)+1}{2} \sum_{k=0}^{N} F_{j}\left(z\left(-1, \widetilde{\vartheta}\left(x_{i}^{\mu}\right), v_{k}\right)\right) \omega_{k}$.

Plugging the second equation in (23) into the first one we obtain

$$
\left\{\begin{aligned}
U^{\prime(\mu)}=\Phi_{1}^{(\mu)}+\frac{\eta_{\mu+1}-\eta_{\mu}}{2} & \left(A^{(\mu)}+R_{1}^{(\mu)}\right) R_{3}^{(\mu)} U^{\prime \mu} \\
& \quad+\left(A^{(\mu)}+R_{1}^{(\mu)}\right) \Phi_{2}^{(\mu)}+B^{(\mu)} V^{(\mu)}, \\
V^{(\mu)}=\Phi_{3}^{(\mu)}+R_{4}^{(\mu)} U^{\prime \mu-1} . &
\end{aligned}\right.
$$

This discrete system is based on the interval $\left[\eta_{\mu}, \eta_{\mu+1}\right]$. The existence of the solution to (24) depends on the existence of the solution to the first matrix equation of (24). Since $A(t), R_{1}(x, z), F_{j}(z)$ are continuous on their definition domains, the elements of the matrices $A^{(\mu)}, R_{1}^{(\mu)}$ and $R_{3}^{(\mu)}, \mu=0,1, \ldots, M$, are all bounded. The Neumann lemma (see [35, p. 26] or [13, p. 87]) then shows that the inverse of the matrix

$$
\mathscr{B}^{(\mu)}:=I-\frac{\eta_{\mu+1}-\eta_{\mu}}{2}\left(A^{(\mu)}+R_{1}^{(\mu)}\right) R_{3}^{(\mu)}
$$

exists whenever

$$
\frac{\eta_{\mu+1}-\eta_{\mu}}{2}\left\|\left(A^{(\mu)}+R_{1}^{(\mu)}\right) R_{3}^{(\mu)}\right\|<1
$$

for some matrix norm. This clearly holds whenever $\eta_{\mu+1}-\eta_{\mu}$ is sufficiently small. For this aim, we divide the interval $\left[\eta_{\mu}, \eta_{\mu+1}\right]$ into $M_{\mu}+1$ subintervals $\left[\tau_{i}^{\mu}, \tau_{i+1}^{\mu}\right] \subseteq\left[\eta_{\mu}, \eta_{\mu+1}\right], i=0,1, \ldots, M_{\mu}, \tau_{0}^{\mu}=\eta_{\mu}, \tau_{M_{\mu}+1}^{\mu}=\eta_{\mu+1}$. The exact solution of (1) still possesses continuous derivatives of order $m+1$ on the subinterval $\left[\tau_{i}^{\mu}, \tau_{i+1}^{\mu}\right], i=0,1, \ldots, M_{\mu}, \mu=0,1, \ldots, M$. Applying the method in Section 2 we use Legendre spectral-collocation method to approximate the exact solution in the basic subinterval $\left[\tau_{i}^{\mu}, \tau_{i+1}^{\mu}\right]$.

Observing each step of the proof for convergence analysis in Section 5, we conclude that the numerical errors decay at an exponential rate no matter how many basic subintervals $\left[\tau_{i}^{\mu}, \tau_{i+1}^{\mu}\right], i=0,1, \ldots, M_{\mu}, \mu=0,1, \ldots, M$, we divide the interval $[-1,1]$ into. Therefore there exists a constant $h_{0}$ such that, for all $\left[\tau_{i}^{\mu}, \tau_{i+1}^{\mu}\right]$ with $\tau_{i+1}^{\mu}-\tau_{i}^{\mu}<h_{0}$, each matrix $\mathscr{B}^{(i \mu)}:=I-\frac{\tau_{i+1}^{\mu}-\tau_{i}^{\mu}}{2}\left(A^{(i \mu)}+R_{1}^{(i \mu)}\right) R_{3}^{(i \mu)}, \quad i=0,1, \ldots, M_{\mu}, \mu=0,1, \ldots, M$, 
has a uniformly bounded inverse. This ensures the corresponding discrete system based on the interval $\left[\tau_{i}^{\mu}, \tau_{i+1}^{\mu}\right]$ possesses a unique solution.

\section{Some useful lemmas}

In this section, we will provide some elementary lemmas, which are important for the derivation of error estimates in Section 5. In order to give the subsequent lemmas conveniently, we first introduce some spaces. For simplicity, we denote by $\partial_{x}^{k} u(x)$ the $k$-th derivative of $u$; i.e., $\partial_{x}^{k} u(x):=\left(d^{k} u / d x^{k}\right)(x)$.

Let $(a, b)$ be a bounded interval of the real line. We denote by $L^{2}(a, b)$ the space of measurable functions $u:(a, b) \rightarrow \mathbb{R}$ such that $\int_{a}^{b}|u(x)|^{2} d x<+\infty$. It is a Hilbert space for the inner product

$$
(u, v):=\int_{a}^{b} u(x) v(x) d x,
$$

which induces the norm

$$
\|v\|_{L^{2}(a, b)}:=\left(\int_{a}^{b}|v(x)|^{2} d x\right)^{1 / 2} .
$$

Let $m \geq 1$ be an integer. We define $H^{m}(a, b)$ to be the vector space of the functions $v \in L^{2}(a, b)$ such that all the distributions of $v$ of order up to $m$ can be represented by functions in $L^{2}(a, b)$. In short,

$$
H^{m}(a, b):=\left\{v \in L^{2}(a, b): \text { for } 0 \leq k \leq m, \partial_{x}^{k} v(x) \in L^{2}(a, b)\right\} .
$$

$H^{m}(a, b)$ is endowed with the inner product

$$
(u, v)_{m}=\sum_{k=0}^{m} \int_{a}^{b} \partial_{x}^{k} u(x) \partial_{x}^{k} v(x) d x
$$

for which $H^{m}(a, b)$ is a Hilbert space. The associated norm is

$$
\|v\|_{H^{m}(a, b)}:=\left((v, v)_{m}\right)^{1 / 2} .
$$

In bounding the approximation error from above, only some of the $L^{2}$ norms appearing on the right-hand side of the above norm enter into play. Thus, for a nonnegative integer $N$, it is convenient to introduce the seminorm

$$
|v|_{H^{m ; N}(a, b)}:=\left(\sum_{k=\min (m, N+1)}^{m}\left\|\partial_{x}^{k} v(x)\right\|_{L^{2}(a, b)}^{2}\right)^{1 / 2},
$$

which implies that if $N \geq m-1$ then $|v|_{H^{m ; N}(a, b)}=\left\|\partial_{x}^{m} v\right\|_{L^{2}(a, b)}$.

Let $\Lambda_{h}$ denote the collection of subintervals $\delta_{\mu}, \mu=0,1, \ldots, M$. Referring to [25], we define the broken Sobolev space $H^{m}\left(\Lambda_{h}\right)$ as 


$$
H^{m}\left(\Lambda_{h}\right):=\left\{u:\left.u\right|_{\delta_{\mu}} \in H^{m}\left(\delta_{\mu}\right), \mu=0,1, \ldots, M\right\} .
$$

The associated norm is

$$
\|u\|_{H^{m}\left(\Lambda_{h}\right)}:=\left(\sum_{k=0}^{m}\left\|u^{(k)}\right\|_{L^{2}\left(\Lambda_{h}\right)}^{2}\right)^{1 / 2},
$$

where

$$
\left\|u^{(k)}\right\|_{L^{2}\left(\Lambda_{h}\right)}^{2}:=\sum_{\mu=0}^{M}\left\|\partial_{x}^{k}\left(\left.u\right|_{\sigma_{\mu}}\right)\right\|_{L^{2}\left(\delta_{\mu}\right)}^{2}, \quad k=0,1, \ldots, m .
$$

For a nonnegative integer $N$, the associated seminorm is

$$
|u|_{H^{m ; N}\left(\Lambda_{h}\right)}:=\left(\sum_{k=\min (m, N+1)}^{m}\left\|u^{(k)}\right\|_{L^{2}\left(\Lambda_{h}\right)}^{2}\right)^{1 / 2} .
$$

If $N \geq m-1$ then $|u|_{H^{m ; N}\left(\Lambda_{h}\right)}=\left\|u^{(m)}\right\|_{L^{2}\left(\Lambda_{h}\right)}$.

The space $L^{\infty}(a, b)$ is the Banach space of measurable functions $u$ that are bounded outside a set of measure zero, equipped with the norm

$$
\|u\|_{L^{\infty}(a, b)}:=\underset{x \in(a, b)}{\operatorname{ess} \sup }|u(x)| .
$$

We denote by $C([a, b])$ the space of continuous functions on the interval $[a, b]$.

We define an interpolation operator $I_{N}$ associated with the collocation points $X_{N}$ as follows: for any continuous function $v \in C([-1,1])$,

$$
I_{N} v(x):=I_{N}^{\mu}\left(\left.v\right|_{\delta_{\mu}}\right)(x) \text { if } x \in\left(\eta_{\mu}, \eta_{\mu+1}\right], 0 \leq \mu \leq M,
$$

where $\left.v\right|_{\delta_{\mu}}(x)$ is the restriction of $v(x)$ to the subinterval $\left[\eta_{\mu}, \eta_{\mu+1}\right]$, and $I_{N}^{\mu}$ is the interpolation operator associated with the collocation points $X^{\mu}$ in the subinterval $\left[\eta_{\mu}, \eta_{\mu+1}\right] ;$ i.e.,

$$
I_{N}^{\mu}\left(\left.v\right|_{\delta_{\mu}}\right)(x):=\left.\sum_{j=0}^{N} v\right|_{\delta_{\mu}}\left(x_{j}^{\mu}\right) F_{j}^{\mu}(x), \quad x \in\left[\eta_{\mu}, \eta_{\mu+1}\right] .
$$

Hereafter, $C$ denotes a generic positive constant that is independent of $N$.

Lemma 1. Assume that $u \in H^{m}(-1,1), m \geq 1, v(x)$ is a bounded function. Then there exists a constant $C$ independent of $u$ and $v$ such that, for $N \geq m-1$,

$$
\begin{aligned}
\left\|u-J_{N} u\right\|_{L^{2}(-1,1)} & \leq C N^{-m}\left\|\partial_{x}^{m} u\right\|_{L^{2}(-1,1)}, \\
\left\|u-J_{N} u\right\|_{L^{\infty}(-1,1)} & \leq C N^{1 / 2-m}\left\|\partial_{x}^{m} u\right\|_{L^{2}(-1,1)}, \\
\sup _{N}\left\|J_{N} v\right\|_{L^{2}(-1,1)} & \leq C\|v\|_{L^{\infty}(-1,1)}, \\
\left\|J_{N}\right\|_{L^{\infty}(-1,1)} & \leq(2 / \pi) \log (N+1)+0.685,
\end{aligned}
$$

where $J_{N}$ is the interpolation operator associated with the $N+1$ Legendre GaussLobatto points in the interval $[-1,1]$. 
Proof. Inequalities (26) and (28) can be found in [15;33; 43], and (29) can be found in [24]. We only prove (27). Using the Sobolev inequality [15, p. 490], we have

$$
\left\|u-J_{N} u\right\|_{L^{\infty}(-1,1)} \leq C\left\|u-J_{N} u\right\|_{L^{2}(-1,1)}^{1 / 2}\left\|u-J_{N} u\right\|_{H^{1}(-1,1)}^{1 / 2} .
$$

Applying the result (26) to $\left\|u-J_{N} u\right\|_{L^{2}(-1,1)}^{1 / 2}$ makes the above inequality become

$$
\left\|u-J_{N} u\right\|_{L^{\infty}(-1,1)} \leq C N^{-m / 2}\left\|\partial_{x}^{m} u\right\|_{L^{2}(-1,1)}^{1 / 2}\left\|u-J_{N} u\right\|_{H^{1}(-1,1)}^{1 / 2},
$$

which leads to (27) because $\left\|u-J_{N} u\right\|_{H^{1}(-1,1)}^{1 / 2}$ can be estimated as follows [15, p. 289]:

$$
\left\|u-J_{N} u\right\|_{H^{1}(-1,1)}^{1 / 2} \leq C N^{(1-m) / 2}\left\|\partial_{x}^{m} u\right\|_{L^{2}(-1,1)}^{1 / 2} .
$$

Lemma 2. Assume that $u \in C([-1,1]) \cap H^{m}\left(\Lambda_{h}\right)$. Let $I_{N} u$ be the interpolation function defined in (25) where $N+1$ is the number of collocation points in the intervals $\left[\eta_{\mu}, \eta_{\mu+1}\right], \mu=0,1, \ldots, M$. Then the following estimates hold for $N \geq m-1$ :

$$
\begin{aligned}
\left\|u-I_{N} u\right\|_{L^{2}(-1,1)} & \leq C N^{-m}\left\|u^{(m)}\right\|_{L^{2}\left(\Lambda_{h}\right)}, \\
\left\|u-I_{N} u\right\|_{L^{\infty}(-1,1)} & \leq C N^{1 / 2-m}\left\|u^{(m)}\right\|_{L^{2}\left(\Lambda_{h}\right)}, \\
\left\|I_{N}\right\|_{L^{\infty}(-1,1)} & \leq C \log (N+1), \\
\sup _{N}\left\|I_{N} u\right\|_{L^{2}(-1,1)} & \leq C\|u\|_{L^{\infty}(-1,1) .}
\end{aligned}
$$

Proof. By the definition of $I_{N}^{\mu}$ we know that the $\left(I_{N}^{\mu}\left(\left.u\right|_{\delta_{\mu}}\right)\right)(z)$ is a function defined on the subinterval $\left[\eta_{\mu}, \eta_{\mu+1}\right]$. The variable transformation $z=z_{\mu}(v)$ changes it to be a function valued on the standard interval $[-1,1]$; i.e., for $v \in[-1,1]$,

$$
\left(I_{N}^{\mu}\left(\left.u\right|_{\delta_{\mu}}\right)\right)\left(z_{\mu}(v)\right)=\left.\sum_{j=0}^{N} u\right|_{\delta_{\mu}}\left(x_{j}^{\mu}\right) F_{j}^{\mu}\left(z_{\mu}(v)\right)=\left.\sum_{j=0}^{N} u\right|_{\delta_{\mu}}\left(x_{j}^{\mu}\right) F_{j}(v) .
$$

The result (16) is used in the derivation of the second equality above. On the other hand, we note that $\left.u\right|_{\delta_{\mu}}\left(z_{\mu}(v)\right)$ is a function defined on the interval $[-1,1]$. Its interpolation polynomial associated with the Legendre Gauss-Lobatto points $v_{j}$, $j=0,1, \ldots, N$, in the interval $[-1,1]$ is

$$
J_{N}\left(\left.u\right|_{\delta_{\mu}}\left(z_{\mu}(v)\right)\right)=\left.\sum_{j=0}^{N} u\right|_{\delta_{\mu}}\left(z_{\mu}\left(v_{j}\right)\right) F_{j}(v), \quad v \in[-1,1] .
$$

Note that $v_{j}=x_{j}$; then

$$
z_{\mu}\left(v_{j}\right)=x_{j}^{\mu}, \quad j=0,1, \ldots, N .
$$

Plugging this into the right-hand side of (36) yields 


$$
J_{N}\left(\left.u\right|_{\delta_{\mu}}\left(z_{\mu}(v)\right)\right)=\left.\sum_{j=0}^{N} u\right|_{\delta_{\mu}}\left(x_{j}^{\mu}\right) F_{j}(v), \quad v \in[-1,1] .
$$

Combining (35) with (37) yields

$$
\left(I_{N}^{\mu}\left(\left.u\right|_{\delta_{\mu}}\right)\right)\left(z_{\mu}(v)\right)=J_{N}\left(\left.u\right|_{\delta_{\mu}}\left(z_{\mu}(v)\right)\right), \quad v \in[-1,1] .
$$

By (26), we have

$$
\begin{aligned}
\int_{\eta_{\mu}}^{\eta_{\mu+1}}\left(\left.u\right|_{\delta_{\mu}}(z)-I_{N}^{\mu}\left(\left.u\right|_{\delta_{\mu}}\right)\right. & (z))^{2} d z \\
& =\frac{\eta_{\mu+1}-\eta_{\mu}}{2} \int_{-1}^{1}\left(\left.u\right|_{\delta_{\mu}}\left(z_{\mu}(v)\right)-J_{N}\left(\left.u\right|_{\delta_{\mu}}\left(z_{\mu}(v)\right)\right)\right)^{2} d v \\
& \leq C N^{-2 m}\left(\frac{\eta_{\mu+1}-\eta_{\mu}}{2}\right)^{2 m+1}\left\|\partial_{v}^{m}\left(\left.u\right|_{\delta_{\mu}}\left(z_{\mu}(\cdot)\right)\right)\right\|_{L^{2}(-1,1)}^{2} \\
& \leq C N^{-2 m}\left\|\partial_{z}^{m}\left(\left.u\right|_{\delta_{\mu}}(\cdot)\right)\right\|_{L^{2}\left(\delta_{\mu}\right)}^{2} .
\end{aligned}
$$

This helps to deduce that

$$
\begin{aligned}
\left\|u-I_{N} u\right\|_{L^{2}(-1,1)}^{2} & =\sum_{\mu=0}^{M} \int_{\eta_{\mu}}^{\eta_{\mu+1}}\left(\left.u\right|_{\delta_{\mu}}(z)-I_{N}^{\mu}\left(\left.u\right|_{\delta_{\mu}}\right)(z)\right)^{2} d z \\
& \leq C N^{-2 m} \sum_{\mu=0}^{M}\left\|\partial_{z}^{m}\left(\left.u\right|_{\delta_{\mu}}(\cdot)\right)\right\|_{L^{2}\left(\delta_{\mu}\right)}^{2}=C N^{-2 m}\left\|u^{(m)}\right\|_{L^{2}\left(\Lambda_{h}\right)}^{2},
\end{aligned}
$$

which leads to (31).

Using (27), we have

$$
\begin{aligned}
\left\|u-I_{N} u\right\|_{L^{\infty}(-1,1)} & =\max _{0 \leq \mu \leq M}\left\{\left\|\left.u\right|_{\delta_{\mu}}\left(z_{\mu}(\cdot)\right)-J_{N}\left(\left.u\right|_{\delta_{\mu}}\left(z_{\mu}(\cdot)\right)\right)\right\|_{L^{\infty}(-1,1)}\right\} \\
& \leq C N^{1 / 2-m} \max _{0 \leq \mu \leq M}\left\{\left\|\partial_{v}^{m}\left(\left.u\right|_{\delta_{\mu}}\left(z_{\mu}(\cdot)\right)\right)\right\|_{L^{2}(-1,1)}\right\} \\
& \leq C N^{1 / 2-m}\left\|u^{(m)}\right\|_{L^{2}\left(\Lambda_{h}\right)} .
\end{aligned}
$$

This is (32).

Now we begin to prove (33). It is evident that

$$
\left\|I_{N} u\right\|_{L^{\infty}(-1,1)}=\max _{0 \leq \mu \leq M}\left\|I_{N}^{\mu}\left(\left.u\right|_{\delta_{\mu}}\right)\right\|_{L^{\infty}\left(\sigma_{\mu}\right)} .
$$

We use (29) to estimate $\left\|I_{N}^{\mu}\left(\left.u\right|_{\delta_{\mu}}\right)\right\|_{L^{\infty}\left(\delta_{\mu}\right)}$ :

$$
\begin{aligned}
\left\|I_{N}^{\mu}\left(\left.u\right|_{\delta_{\mu}}\right)\right\|_{L^{\infty}\left(\delta_{\mu}\right)} & =\left\|\left(I_{N}^{\mu}\left(\left.u\right|_{\delta_{\mu}}\right)\right)\left(z_{\mu}(\cdot)\right)\right\|_{L^{\infty}(-1,1)}=\left\|J_{N}\left(\left.u\right|_{\delta_{\mu}}\left(z_{\mu}(\cdot)\right)\right)\right\|_{L^{\infty}(-1,1)} \\
& \leq C \log (N+1)\left\|\left.u\right|_{\delta_{\mu}}\left(z_{\mu}(\cdot)\right)\right\|_{L^{\infty}(-1,1)}=C \log (N+1)\left\|\left.u\right|_{\delta_{\mu}}\right\|_{L^{\infty}\left(\delta_{\mu}\right)} \\
& \leq C \log (N+1)\|u\|_{L^{\infty}(-1,1)},
\end{aligned}
$$


which together with (42) give that

$$
\left\|I_{N} u\right\|_{L^{\infty}(-1,1)} \leq C \log (N+1)\|u\|_{L^{\infty}(-1,1)} .
$$

This leads to the desired result (33).

Now we begin to prove (34). The result (28) is useful in the following derivation:

$$
\begin{aligned}
\left\|I_{N} u\right\|_{L^{2}(-1,1)}^{2} & =\sum_{\mu=0}^{M}\left\|I_{N}^{\mu}\left(\left.u\right|_{\delta_{\mu}}\right)\right\|_{L^{2}\left(\delta_{\mu}\right)}^{2}=\sum_{\mu=0}^{M} \frac{\eta_{\mu+1}-\eta_{\mu}}{2}\left\|\left(I_{N}^{\mu}\left(\left.u\right|_{\delta_{\mu}}\right)\right)\left(z_{\mu}(\cdot)\right)\right\|_{L^{2}(-1,1)}^{2} \\
& =\sum_{\mu=0}^{M} \frac{\eta_{\mu+1}-\eta_{\mu}}{2}\left\|J_{N}\left(\left.u\right|_{\delta_{\mu}}\left(z_{\mu}(\cdot)\right)\right)\right\|_{L^{2}(-1,1)}^{2} \\
& \leq C \sum_{\mu=0}^{M} \frac{\eta_{\mu+1}-\eta_{\mu}}{2}\left\|\left.u\right|_{\delta_{\mu}}\left(z_{\mu}(\cdot)\right)\right\|_{L^{\infty}(-1,1)}^{2} \\
& \leq C \sum_{\mu=0}^{M} \frac{\eta_{\mu+1}-\eta_{\mu}}{2}\|u\|_{L^{\infty}(-1,1)}^{2} \leq C\|u\|_{L^{\infty}(-1,1)}^{2}
\end{aligned}
$$

which leads to the desired result (34). Now we have completed the whole proof for this lemma.

Lemma 3 [15; 41]. Assume that $u \in H^{m}(-1,1)$ for some $m \geq 1$ and $\varphi \in \mathscr{P}_{N}$, which denotes the space of all polynomials of degree not exceeding $N$. Then there exists a constant $C$ independent of $N \geq m-1$ such that

$$
\left|\int_{-1}^{1} u(x) \varphi(x) d x-\sum_{j=0}^{N} u\left(x_{j}\right) \varphi\left(x_{j}\right) \omega_{j}\right| \leq C N^{-m}\left\|\partial_{x}^{m} u\right\|_{L^{2}(-1,1)}\|\varphi\|_{L^{2}(-1,1)},
$$

where $x_{j}$ are the $N+1$ Legendre Gauss-Lobatto points, with corresponding weights $\omega_{j}, j=0,1, \ldots, N$.

Lemma 4 [43]. Suppose $0 \leq M<+\infty$. If a nonnegative integrable function $e(x)$ satisfies

$$
e(x) \leq v(x)+M \int_{-1}^{x} e(z) d z
$$

where $v(x)$ is also a nonnegative integrable function, then

$$
\|e(x)\|_{L^{p}(-1,1)} \leq C\|v(x)\|_{L^{p}(-1,1)}, \quad p=2,+\infty .
$$

\section{Convergence analysis}

This section is devoted to providing a convergence analysis for the numerical scheme. The goal is to show that the rate of convergence is exponential; i.e., the spectral 
accuracy can be obtained for the proposed approximations. Firstly, we will carry out convergence analysis in the $L^{\infty}(-1,1)$ space.

Theorem 1. Let $u(x)$ be the exact solution to $(5), u^{N}(x)$ be the approximate solution, and $\rho^{N}(x)$ be the approximate derivative obtained by using the spectralcollocation schemes (17), (19) and (22). Then, for $N \geq m-1$ sufficiently large,

$$
\left\|e_{i}(x)\right\|_{L^{\infty}(-1,1)} \leq C N^{1 / 2-m}\left(R\|u\|_{L^{\infty}(-1,1)}+\left\|u^{(m+1)}\right\|_{L^{2}\left(\Lambda_{h}\right)}\right), \quad i=0,1,
$$

where

$$
\begin{aligned}
& e_{0}(x):=\left\{\begin{array}{cl}
0, & x \in[\vartheta(-1),-1], \\
u(x)-u^{N}(x), & x \in(-1,1],
\end{array}\right. \\
& e_{1}(x):=\left\{\begin{array}{cl}
0, & x \in[\vartheta(-1),-1], \\
u^{\prime}(x)-\rho^{N}(x), & x \in(-1,1],
\end{array}\right.
\end{aligned}
$$

and $R$ is a constant dependent on the $m$-order derivatives of $R_{j}(x, z), \psi(z), j=1,2$.

Proof. In each subinterval $\left(\eta_{\mu}, \eta_{\mu+1}\right], \mu=0,1, \ldots, M$, the degree of the polynomial $\rho^{N}(s)$ does not exceed $N$. Then

$$
\alpha_{1}\left(x_{i}^{\mu}\right)=\int_{-1}^{x_{i}^{\mu}} \rho^{N}(z) d z \quad \text { and } \quad \alpha_{2}\left(x_{i}^{\mu}\right)=\int_{-1}^{\vartheta\left(x_{i}^{\mu}\right)} \rho^{N}(z) d z
$$

which implies that

$$
\begin{gathered}
u\left(x_{i}^{\mu}\right)-u_{i}^{\mu}=\int_{-1,}^{x_{i}^{\mu}} e_{1}(z) d z, \\
u\left(\vartheta\left(x_{i}^{\mu}\right)\right)-v_{i}^{\mu}=\int_{-1,}^{\vartheta\left(x_{i}^{\mu}\right)} e_{1}(z) d z .
\end{gathered}
$$

Subtracting (17) from (9) yields

$$
\begin{aligned}
u^{\prime}\left(x_{i}^{\mu}\right)-\rho_{i}^{\mu}=A\left(x_{i}^{\mu}\right) \int_{-1}^{x_{i}^{\mu}} e_{1}(z) d z & +B\left(x_{i}^{\mu}\right) \int_{-1}^{\vartheta\left(x_{i}^{\mu}\right)} e_{1}(z) d z+\int_{-1}^{x_{i}^{\mu}} R_{1}\left(x_{i}^{\mu}, z\right) e_{0}(z) d z \\
+ & \int_{-1}^{\vartheta\left(x_{i}^{\mu}\right)} R_{2}\left(x_{i}^{\mu}, z\right) e_{0}(z) d z+\sum_{j=0}^{1} E_{j}\left(x_{i}^{\mu}\right),
\end{aligned}
$$

where, for $x \in[-1,1]$,

$$
E_{1}(x):=\int_{-1}^{x} R_{1}(x, z) u^{N}(z) d z-\beta(x), \quad E_{0}(x):=\int_{-1}^{\vartheta(x)} R_{2}(x, z) u^{N}(z) d z-\lambda(x),
$$


Multiplying by $F_{i}^{\mu}(x)$ on both sides of (48) and summing from $i=0$ to $N$, we obtain that

$$
\begin{aligned}
& \sum_{i=0}^{N} u^{\prime}\left(x_{i}^{\mu}\right) F_{i}^{\mu}(x)-\sum_{i=0}^{N} \rho_{i}^{\mu} F_{i}^{\mu}(x) \\
& =\sum_{i=0}^{N}\left(A\left(x_{i}^{\mu}\right) \int_{-1}^{x_{i}^{\mu}} e_{1}(z) d z\right) F_{i}^{\mu}(x)+\sum_{i=0}^{N}\left(B\left(x_{i}^{\mu}\right) \int_{-1}^{\vartheta\left(x_{i}^{\mu}\right)} e_{1}(z) d z\right) F_{i}^{\mu}(x) \\
& \quad+\sum_{i=0}^{N}\left(\int_{-1}^{x_{i}^{\mu}} R_{1}\left(x_{i}^{\mu}, z\right) e_{0}(z) d z\right) F_{i}^{\mu}(x)+\sum_{i=0}^{N}\left(\int_{-1}^{\vartheta\left(x_{i}^{\mu}\right)} R_{2}\left(x_{i}^{\mu}, z\right) e_{0}(z) d z\right) F_{i}^{\mu}(x) \\
& \quad+\sum_{j=0}^{1} \sum_{i=0}^{N} E_{j}\left(x_{i}^{\mu}\right) F_{i}^{\mu}(x), \quad x \in\left[\eta_{\mu}, \eta_{\mu+1}\right] .
\end{aligned}
$$

By the definitions of $I_{N}$ and $\rho^{N}(x)$, we have

$$
\begin{aligned}
I_{N} u^{\prime}(x)-\rho^{N}(x)= & I_{N}\left(A(x) \int_{-1}^{x} e_{1}(z) d z\right)+I_{N}\left(B(x) \int_{-1}^{\vartheta(x)} e_{1}(z) d z\right) \\
& +I_{N}\left(\int_{-1}^{x} R_{1}(x, z) e_{0}(z) d z\right)+I_{N}\left(\int_{-1}^{\vartheta(x)} R_{2}(x, z) e_{0}(z) d z\right) \\
& +\sum_{j=0}^{1} I_{N} E_{j}(x), \quad x \in[-1,1] .
\end{aligned}
$$

This leads to

$$
\begin{aligned}
e_{1}(x)=\sum_{j=0}^{1} I_{N} E_{j}(x)+ & \sum_{j=2}^{6} E_{j}(x)+A(x) \int_{-1}^{x} e_{1}(z) d z+B(x) \int_{-1}^{\vartheta(x)} e_{1}(z) d z \\
& +\int_{-1}^{x} R_{1}(x, z) e_{0}(z) d z+\int_{-1}^{\vartheta(x)} R_{2}(x, z) e_{0}(z) d z
\end{aligned}
$$

where

$$
\begin{aligned}
& E_{2}(x):=\left(I-I_{N}\right) u^{\prime}(x), \\
& E_{3}(x):=\left(I_{N}-I\right) \int_{-1}^{x} R_{1}(x, z) e_{0}(z) d z, \\
& E_{4}(x):=\left(I_{N}-I\right) \int_{-1}^{\vartheta(x)} R_{2}(x, z) e_{0}(z) d z, \\
& E_{5}(x):=\left(I_{N}-I\right)\left(A(x) \int_{-1}^{x} e_{1}(z) d z\right), \\
& E_{6}(x):=\left(I_{N}-I\right)\left(B(x) \int_{-1}^{\vartheta(x)} e_{1}(z) d z\right) .
\end{aligned}
$$


Applying the Dirichlet formula to the last two terms in the right-hand side of (51) yields

$$
\begin{aligned}
\int_{-1}^{x} R_{1}(x, z) e_{0}(z) d z & =\int_{-1}^{x}\left[\int_{s}^{x} R_{1}(x, z) d z\right] e_{1}(s) d s, \\
\int_{-1}^{\vartheta(x)} R_{2}(x, z) e_{0}(z) d z & =\int_{-1}^{\vartheta(x)}\left[\int_{s}^{\vartheta(x)} R_{2}(x, z) d z\right] e_{1}(s) d s,
\end{aligned}
$$

which help to deduce that there exist constants $C_{1}, C_{2}, C>0$ such that

$$
\begin{aligned}
& \mid A(x) \int_{-1}^{x} e_{1}(z) d z+ B(x) \int_{-1}^{\vartheta(x)} e_{1}(z) d z+\int_{-1}^{x} R_{1}(x, z) e_{0}(z) d z+\int_{-1}^{\vartheta(x)} R_{2}(x, z) e_{0}(z) d z \mid \\
& \leq C_{1} \int_{-1}^{x} e_{1}(z) d z+C_{2} \int_{-1}^{\vartheta(x)} e_{1}(z) d z \leq C \int_{-1}^{x} e_{1}(z) d z
\end{aligned}
$$

Then, by Lemma $4, e_{1}(x)$ in (51) can be estimated as follows:

$$
\left\|e_{1}(x)\right\|_{L^{\infty}(-1,1)} \leq C\left(\sum_{j=0}^{1}\left\|I_{N} E_{j}(x)\right\|_{L^{\infty}(-1,1)}+\sum_{j=2}^{6}\left\|E_{j}(x)\right\|_{L^{\infty}(-1,1)}\right) .
$$

We estimate each term of the right-hand side of the above inequality one by one.

First we estimate $\left\|I_{N} E_{0}(x)\right\|_{L^{\infty}(-1,1)}$. By (33) we have

$$
\left\|I_{N} E_{0}(x)\right\|_{L^{\infty}(-1,1)} \leq C \log (N+1)\left\|E_{0}(x)\right\|_{L^{\infty}(-1,1)} .
$$

We estimate $\left\|E_{0}(x)\right\|_{L^{\infty}(-1,1)}$. Note that $E_{0}(x)$ can be written as

$$
E_{0}(x)=\left\{\begin{array}{l}
\int_{-1}^{\vartheta(x)} R_{2}(x, z) \psi(z) d z-\frac{\vartheta(x)+1}{2} \lambda_{2}(x), \quad x \in \delta_{0}, \\
\sum_{r=0}^{\mu-2}\left(\int_{\eta_{r}}^{\eta_{r+1}} R_{2}(x, z) u_{r}(z) d z-\frac{\eta_{r+1}-\eta_{r}}{2} \lambda_{1}^{r}(x)\right) \\
\quad+\int_{\eta_{\mu-1}}^{\vartheta(x)} R_{2}(x, z) u_{\mu-1}(z) d z-\frac{\eta_{\mu}-\eta_{\mu-1}}{2} \frac{\tilde{\vartheta}(x)+1}{2} \lambda_{3}(x), \\
x \in \delta_{\mu}, \mu>0 .
\end{array}\right.
$$

Lemma 3 helps to deduce that

$$
\begin{aligned}
\left|\int_{-1}^{\vartheta(x)} R_{2}(x, z) \psi(z) d z-\frac{\vartheta(x)+1}{2} \lambda_{2}(x)\right| \\
\leq C N^{-m}\left\|\partial_{v}^{m}\left(R_{2}(x, z(-1, \vartheta(x), \cdot)) \psi(z(-1, \vartheta(x), \cdot))\right)\right\|_{L^{2}(-1,1)} \\
\leq C N^{-m}\left|\frac{\vartheta(x)+1}{2}\right|^{m}\left\|\left.\partial_{z}^{m}\left(R_{2}(x, z) \psi(z)\right)\right|_{z=z(-1, \vartheta(x), \cdot)}\right\|_{L^{2}(-1,1)} \\
\leq C N^{-m}\left\|\partial_{z}^{m}\left(R_{2}(x, \cdot) \psi(\cdot)\right)\right\|_{L^{2}(\vartheta(x),-1)}, \quad x \in \delta_{0},
\end{aligned}
$$


and, for $x \in \delta_{\mu}, \mu>0$,

$$
\begin{aligned}
& \left|\int_{\eta_{r}}^{\eta_{r+1}} R_{2}(x, z) u_{r}(z) d z-\frac{\eta_{r+1}-\eta_{r}}{2} \lambda_{1}^{r}(x)\right| \\
& \quad \leq C N^{-m}\left|\frac{\eta_{r+1}-\eta_{r}}{2}\right|\left\|\partial_{v}^{m}\left(R_{2}\left(x, z_{r}(x, \cdot)\right)\right)\right\|_{L^{2}(-1,1)}\left\|u_{r}\left(z_{r}(\cdot)\right)\right\|_{L^{2}(-1,1)} \\
& \quad \leq C N^{-m}\left|\frac{\eta_{r+1}-\eta_{r}}{2}\right|^{m+1 / 2}\left\|\left.\partial_{z}^{m}\left(R_{2}(x, z)\right)\right|_{z=z_{r}(\cdot)}\right\|_{L^{2}(-1,1)}\left\|u_{r}\right\|_{L^{2}\left(\delta_{r}\right)} \\
& \quad \leq C N^{-m}\left\|\partial_{z}^{m}\left(R_{2}(x, \cdot)\right)\right\|_{L^{2}\left(\delta_{r}\right)}\left\|u_{r}\right\|_{L^{2}\left(\delta_{r}\right)} .
\end{aligned}
$$

Similarly,

$$
\begin{aligned}
& \mid \int_{\eta_{\mu-1}}^{\vartheta(x)} R_{2}(x, z) u_{\mu-1}(z) d z-\frac{\eta_{\mu}-\eta_{\mu-1}}{2} \frac{\widetilde{\vartheta}_{\mu-1}(x)+1}{2} \lambda_{3}(x) \mid \\
& \leq C N^{-m}\left\|\partial_{z}^{m}\left(R_{2}(x, \cdot)\right)\right\|_{L^{2}\left(\eta_{\mu-1}, \vartheta(x)\right)}\left\|u_{\mu-1}\right\|_{L^{2}\left(\delta_{\mu-1}\right)}
\end{aligned}
$$

By the Cauchy inequality, which states that

$$
\sum_{r=0}^{\mu-1} a_{r} b_{r} \leq\left(\sum_{r=0}^{\mu-1} a_{r}^{2}\right)^{1 / 2}\left(\sum_{r=0}^{\mu-1} b_{r}^{2}\right)^{1 / 2},
$$

in which we let

$$
\begin{gathered}
a_{r}=\left\|\partial_{z}^{m}\left(R_{2}(x, \cdot)\right)\right\|_{L^{2}\left(\delta_{r}\right)}, \quad b_{r}=\left\|u_{r}\right\|_{L^{2}\left(\delta_{r}\right)}, \quad r=0,1, \ldots, \mu-2, \\
a_{\mu-1}=\left\|\partial_{z}^{m}\left(R_{2}(x, \cdot)\right)\right\|_{L^{2}\left(\eta_{\mu-1}, \vartheta(x)\right)}, \quad b_{\mu-1}=\left\|u_{\mu-1}\right\|_{L^{2}\left(\delta_{\mu-1}\right)}
\end{gathered}
$$

we have, for $x \in \delta_{\mu}, \mu>0$,

$$
\begin{aligned}
\left|E_{0}(x)\right| & \leq C N^{-m}\left\|\partial_{z}^{m}\left(R_{2}(x, \cdot)\right)\right\|_{L^{2}(-1, \vartheta(x))}\left\|u^{N}\right\|_{L^{2}(-1,1)} \\
& \leq C N^{-m}\left\|\partial_{z}^{m}\left(R_{2}(x, \cdot)\right)\right\|_{L^{2}(-1, \vartheta(x))}\left(\left\|e_{0}\right\|_{L^{\infty}(-1,1)}+\|u\|_{L^{\infty}(-1,1)}\right) .
\end{aligned}
$$

Then

$$
\left\|E_{0}(x)\right\|_{L^{\infty}(-1,1)} \leq C N^{-m} \widetilde{R}_{2}\left(\left\|e_{0}\right\|_{L^{\infty}(-1,1)}+\|u\|_{L^{\infty}(-1,1)}\right)
$$

where

$$
\widetilde{R}_{2}:=\max \left\{\max _{x \in \delta_{0}}\left\|\partial_{z}^{m}\left(R_{2}(x, \cdot) \psi(\cdot)\right)\right\|_{L^{2}(\vartheta(x),-1)}, \max _{x \in\left[\eta_{1}, 1\right]}\left\|\partial_{z}^{m}\left(R_{2}(x, \cdot)\right)\right\|_{L^{2}(-1, \vartheta(x))}\right\} .
$$

Therefore, combining (63) with (57) gives

$$
\left\|I_{N} E_{0}(x)\right\|_{L^{\infty}(-1,1)} \leq C N^{-m} \log (N+1) \widetilde{R}_{2}\left(\left\|e_{0}\right\|_{L^{\infty}(-1,1)}+\|u\|_{L^{\infty}(-1,1)}\right) .
$$

Using the same analysis as for $\left\|I_{N} E_{0}(x)\right\|_{L^{\infty}(-1,1)}$, we can obtain the estimate

$$
\left\|I_{N} E_{1}(x)\right\|_{L^{\infty}(-1,1)} \leq C N^{-m} \log (N+1) \widetilde{R}_{1}\left(\left\|e_{0}\right\|_{L^{\infty}(-1,1)}+\|u\|_{L^{\infty}(-1,1)}\right),
$$


where

$$
\widetilde{R}_{1}:=\max _{x \in[-1,1]}\left\|\partial_{z}^{m}\left(R_{1}(x, \cdot)\right)\right\|_{L^{2}(-1, x)} .
$$

Now we begin to estimate $\left\|E_{j}(x)\right\|_{L^{\infty}(-1,1)}, j=2,3,4,5,6$. Note that, in each subinterval $\delta_{\mu}, \mu=0,1, \ldots, M,\left.u^{\prime}\right|_{\delta_{\mu}}(x) \in H^{m}\left(\delta_{\mu}\right)$. Applying (32) to $u^{\prime}(x)$, we have

$$
\left\|E_{2}(x)\right\|_{L^{\infty}(-1,1)}\left\|\leq C N^{1 / 2-m}\right\| u^{(m+1)} \|_{L^{2}\left(\Lambda_{h}\right)} .
$$

Now we begin to estimate $\left\|E_{4}(x)\right\|_{L^{\infty}(-1,1)}$. For simplicity of notation, we set

$$
b(x):=\int_{-1}^{\vartheta(x)} R_{2}(x, z) e_{0}(z) d z
$$

Applying (32) with $m=1$ to $b(x)$ yields

$$
\left\|\left(I_{N}-I\right) b(x)\right\|_{L^{\infty}(-1,1)} \leq C N^{-1 / 2}\left\|\partial_{x}^{1} b\right\|_{L^{2}(-1,1)} .
$$

Note that

$$
\begin{aligned}
\left|\partial_{x}^{1} b(x)\right| & =\left|R_{2}(x, \vartheta(x)) e_{0}(\vartheta(x)) \vartheta^{\prime}(x)+\int_{-1}^{\vartheta(x)} \frac{\partial R_{2}}{\partial x}(x, z) e_{0}(z) d z\right| \\
& \leq\left\|e_{0}\right\|_{L^{\infty}(-1,1)}\left|\left(R_{2}(x, \vartheta(x)) \vartheta^{\prime}(x)+\int_{-1}^{\vartheta(x)} \frac{\partial R_{2}}{\partial x}(x, z) d z\right)\right| \\
& \leq C\left\|e_{0}\right\|_{L^{\infty}(-1,1)},
\end{aligned}
$$

which, together with (67), yields

$$
\left\|E_{4}(x)\right\|_{L^{\infty}(-1,1)}=\left\|\left(I_{N}-I\right) b(x)\right\|_{L^{\infty}(-1,1)} \leq C N^{-1 / 2}\left\|e_{0}\right\|_{L^{\infty}(-1,1)} .
$$

Similarly,

$$
\begin{aligned}
&\left\|E_{3}(x)\right\|_{L^{\infty}(-1,1)} \leq C N^{-1 / 2}\left\|e_{0}\right\|_{L^{\infty}(-1,1)}, \\
&\left\|E_{5}(x)\right\|_{L^{\infty}(-1,1)} \leq C N^{-1 / 2}\left\|e_{1}\right\|_{L^{\infty}(-1,1)}, \\
&\left\|E_{6}(x)\right\|_{L^{\infty}(-1,1)} \leq C N^{-1 / 2}\left\|e_{1}\right\|_{L^{\infty}(-1,1)} .
\end{aligned}
$$

Combining (56) with (64), (65), (66), (69) and (70) yields that

$$
\begin{aligned}
\left\|e_{1}(x)\right\|_{L^{\infty}(-1,1)} \leq C N^{-m}\left(\log (N+1) R\|u\|_{L^{\infty}(-1,1)}\right. & \left.+N^{1 / 2}\left\|u^{(m+1)}\right\|_{L^{2}\left(\Lambda_{h}\right)}\right) \\
& +C N^{-1 / 2}\left\|e_{0}\right\|_{L^{\infty}(-1,1)},
\end{aligned}
$$

where

$$
R:=\max \left\{\widetilde{R}_{1}, \widetilde{R}_{2}\right\}
$$

Now we need another relation between $\left\|e_{1}(x)\right\|_{L^{\infty}(-1,1)}$ and $\left\|e_{0}\right\|_{L^{\infty}(-1,1)}$. Multiplying by $F_{i}^{\mu}(x)$ on both sides of (47) and summing from $i=0$ to $N$ for 
$\mu=0,1, \ldots, M$, we obtain that

$$
e_{0}(x)=E_{7}(x)+\left(I_{N}-I\right)\left(\int_{-1}^{x} e_{1}(s) d s\right)+\int_{-1}^{x} e_{1}(s) d s
$$

where

$$
E_{7}(x):=\left(I-I_{N}\right) u(x) .
$$

Then

$\left\|e_{0}\right\|_{L^{\infty}(-1,1)}$

$$
\leq C\left(\left\|E_{7}(x)\right\|_{L^{\infty}(-1,1)}+\left\|\left(I_{N}-I\right)\left(\int_{-1}^{x} e_{1}(s) d s\right)\right\|_{L^{\infty}(-1,1)}+\left\|e_{1}\right\|_{L^{\infty}(-1,1)}\right) .
$$

Using (32) for $E_{7}(x)$, and applying (32) with $m=1$ to the middle term of the right-hand side of the above inequality, we have

$$
\left\|e_{0}\right\|_{L^{\infty}(-1,1)} \leq C N^{-m-1 / 2}\left\|u^{(m+1)}\right\|_{L^{2}\left(\Lambda_{h}\right)}+C\left\|e_{1}\right\|_{L^{\infty}(-1,1)} .
$$

Plugging the above result into the last term of (71) yields the desired estimate (45) for $e_{1}$, which, in turn, substituted into the last term of (73), yields the estimate (45) for $e_{0}$.

Next, we will give the error estimate in the $L^{2}(-1,1)$ space.

Theorem 2. Let $u(x)$ be the exact solution to $(5)$. Let $u^{N}(x)$ be the approximate solution, and $\rho^{N}(x)$ be the approximate derivative obtained by using the spectralcollocation schemes (17), (19) and (22). Then, for $N \geq m-1$ sufficiently large,

$$
\left\|e_{i}\right\|_{L^{2}(-1,1)} \leq C N^{-m} R(R+1)\left(\|u\|_{L^{\infty}(-1,1)}+\left\|u^{(m+1)}\right\|_{L^{2}\left(\Lambda_{h}\right)}\right), \quad i=0,1 .
$$

Proof. By Lemma 4, it follows from (51) and (55) that

$$
\left\|e_{1}(x)\right\|_{L^{2}(-1,1)} \leq C\left(\sum_{j=0}^{1}\left\|I_{N} E_{j}(x)\right\|_{L^{2}(-1,1)}+\sum_{j=2}^{6}\left\|E_{j}(x)\right\|_{L^{2}(-1,1)}\right) .
$$

We estimate each term on the right of the above inequality one by one. Applying (34) to $E_{0}(x)$ yields

$$
\left\|I_{N} E_{0}(x)\right\|_{L^{2}(-1,1)} \leq C\left\|E_{0}(x)\right\|_{L^{\infty}(-1,1)} .
$$

Recalling the result (63) and using the result of Theorem 1, we obtain that

$$
\left\|I_{N} E_{0}(x)\right\|_{L^{2}(-1,1)} \leq C N^{-m} R(R+1)\left(\|u\|_{L^{\infty}(-1,1)}+\left\|u^{(m+1)}\right\|_{L^{2}\left(\Lambda_{h}\right)}\right) .
$$

Similarly,

$$
\left\|I_{N} E_{1}(x)\right\|_{L^{2}(-1,1)} \leq C N^{-m} R(R+1)\left(\|u\|_{L^{\infty}(-1,1)}+\left\|u^{(m+1)}\right\|_{L^{2}\left(\Lambda_{h}\right)}\right) .
$$


Note that, in each subinterval $\delta_{\mu}, \mu=0,1, \ldots, M,\left.u^{\prime}\right|_{\delta_{\mu}}(x) \in H^{m}\left(\delta_{\mu}\right)$. Applying (31) to $u^{\prime}(x)$, we have

$$
\left\|E_{2}(x)\right\|_{L^{2}(-1,1)} \leq C N^{-m}\left\|u^{(m+1)}\right\|_{L^{2}\left(\Lambda_{h}\right)} .
$$

Applying the analysis from (67)-(69), using (31) in Lemma 2 with $m=1$ for $b(x)$ yields

$$
\left\|E_{4}\right\|_{L^{2}(-1,1)}=\left\|\left(I-I_{N}\right) b(x)\right\|_{L^{2}(-1,1)} \leq C N^{-1}\left\|e_{0}\right\|_{L^{\infty}(-1,1)} .
$$

Using the estimate for $e_{0}$ in Theorem 1 makes the above inequality become

$$
\left\|E_{4}\right\|_{L^{2}(-1,1)} \leq C N^{-m-1 / 2}\left(R\|u\|_{\infty}+\left\|u^{(m+1)}\right\|_{L^{2}\left(\Lambda_{h}\right)}\right) .
$$

Similarly,

$$
\left\|E_{3}\right\|_{L^{2}(-1,1)} \leq C N^{-m-1 / 2}\left(R\|u\|_{\infty}+\left\|u^{(m+1)}\right\|_{L^{2}\left(\Lambda_{h}\right)}\right) .
$$

Using the same analysis from (67)-(69), using (31) in Lemma 2 with $m=1$ we obtain

$$
\left\|E_{i}\right\|_{L^{2}(-1,1)} \leq C N^{-1}\left\|e_{1}\right\|_{L^{\infty}(-1,1)}, \quad i=5,6 .
$$

Combining (75) with (77), (78), (79), (81), (82) and (83) we obtain the estimate (74) for $e_{1}$.

Now we begin to estimate $\left\|e_{0}\right\|_{L^{2}(-1,1)}$. From (72) we have

$\left\|e_{0}\right\|_{L^{2}(-1,1)} \leq C\left(\left\|E_{7}(x)\right\|_{L^{2}(-1,1)}+\left\|\left(I_{N}-I\right) \int_{-1}^{x} e_{1}(s) d s\right\|_{L^{2}(-1,1)}+\left\|e_{1}\right\|_{L^{2}(-1,1)}\right)$.

Using (31) for $E_{7}(x)$, and applying (31) with $m=1$ to the middle term of the right-hand side of the above inequality, we have

$$
\left\|e_{0}\right\|_{L^{2}(-1,1)} \leq C N^{-m-1}\left\|u^{(m+1)}\right\|_{L^{2}\left(\Lambda_{h}\right)}+C\left\|e_{1}\right\|_{L^{2}(-1,1)},
$$

which leads to the estimate (74) for $e_{0}$ by plugging the result (74) for $e_{1}$ into the last term of (84).

\section{Numerical examples}

In this section, we give four numerical examples. The first one is the linear case with smooth solution. The second one is the linear case with solution unsmooth at the primary discontinuous points. The third one is the nonlinear case. The fourth one is the case in which the delay is a function of the solution to the equations. These examples confirm the theoretical results obtained in the previous section. 


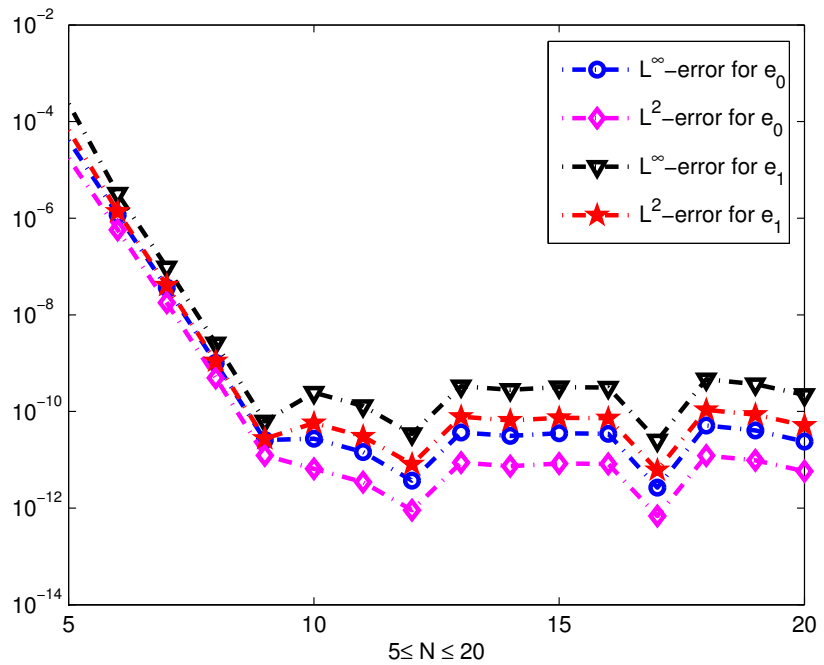

Figure 1. Example 1: Errors versus $N$ in $L^{\infty}$ and $L^{2}$ norms.

Example 1. Consider (1) with

$$
\begin{gathered}
T=4, \quad a(t)=t, \quad b(t)=t^{2}, \quad K_{1}(t, s)=\sin (t+s), \\
K_{2}(t, s)=\cos (t+s), \quad \theta(t)=t-1, \quad \phi(t)=e^{t},
\end{gathered}
$$

$g(t)=e^{t}-t e^{t}-t^{2} e^{t-1}+\sin t-\frac{1}{2}\left[e^{t}(\sin 2 t-\cos 2 t)+e^{t-1}(\cos (2 t-1)+\sin (2 t-1))\right]$.

The corresponding exact solution is $y(t)=e^{t}, t \in(0, T]$.

Figure 1 plots the errors for $5 \leq N \leq 20$ in both $L^{\infty}$ and $L^{2}$ norms. Moreover, the corresponding errors versus several values of $N$ are displayed in Table 1. As expected, the errors decay exponentially, which confirms our theoretical predictions. This example shows that our method is also valid for the nonvanishing delay VIDEs with smooth solution.

Example 2. Consider (1) with

$$
\begin{gathered}
T=3, \quad a(t)=0, \quad b(t)=g(t)=e^{t}, \quad K_{1}(t, s)=0, \\
K_{2}(t, s)=e^{t+s}, \quad \theta(t)=t-\left(\frac{1}{2}+\frac{1}{2} t\right), \quad \phi(t)=1 .
\end{gathered}
$$

\begin{tabular}{rcccccc}
\hline$N$ & 5 & 8 & 11 & 14 & 17 & 20 \\
\hline$L^{\infty}$-error for $e_{0}$ & $4.04 \cdot 10^{-05}$ & $1.01 \cdot 10^{-09}$ & $1.45 \cdot 10^{-11}$ & $3.11 \cdot 10^{-11}$ & $2.66 \cdot 10^{-12}$ & $2.38 \cdot 10^{-11}$ \\
$L^{2}$-error for $e_{0}$ & $1.71 \cdot 10^{-05}$ & $4.92 \cdot 10^{-10}$ & $3.44 \cdot 10^{-12}$ & $7.37 \cdot 10^{-12}$ & $6.87 \cdot 10^{-13}$ & $5.81 \cdot 10^{-12}$ \\
$L^{\infty}$-error for $e_{1}$ & $2.32 \cdot 10^{-04}$ & $2.58 \cdot 10^{-09}$ & $1.30 \cdot 10^{-10}$ & $2.81 \cdot 10^{-10}$ & $2.53 \cdot 10^{-11}$ & $2.20 \cdot 10^{-10}$ \\
$L^{2}$-error for $e_{1}$ & $6.65 \cdot 10^{-05}$ & $1.10 \cdot 10^{-09}$ & $3.03 \cdot 10^{-11}$ & $6.53 \cdot 10^{-11}$ & $6.09 \cdot 10^{-12}$ & $5.12 \cdot 10^{-11}$ \\
\hline
\end{tabular}

Table 1. Example 1: Errors versus $N$ in $L^{\infty}$ and $L^{2}$ norms. 


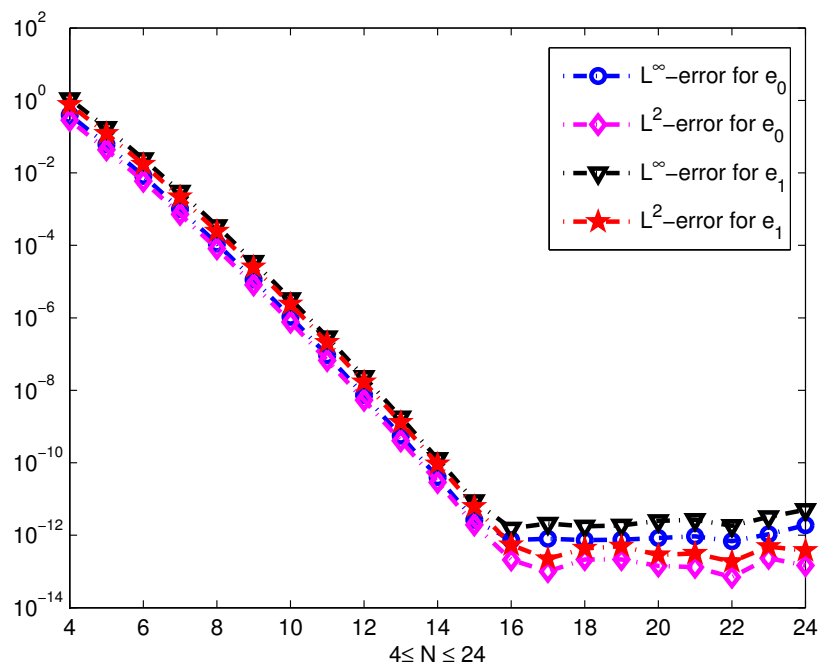

Figure 2. Example 2: Errors versus $N$ in $L^{\infty}$ and $L^{2}$ norms.

The corresponding exact solution is

$$
y(t)=\left\{\begin{array}{cc}
e^{t}+\frac{2}{3} e^{-1 / 2}\left(e^{3 t / 2}-1\right), & t \in(0,1], \\
y_{1}(t), & t \in(1,3],
\end{array}\right.
$$

where

$$
\begin{aligned}
y_{1}(t):=\frac{2}{3} e^{(3 t-1) / 2}+\frac{8}{21} e^{(7 t-5) / 4}+\frac{1}{2} e^{t}+\frac{1}{4} e^{2 t-1}+\frac{16}{135} e^{(9 t-7) / 4} \\
\quad-\frac{4}{15} e^{t-1 / 2}-\frac{4}{9} e^{(3 t-2) / 2}+\frac{1}{4} e-\frac{40}{189} e^{1 / 2}-\frac{2}{3} e^{-1 / 2} .
\end{aligned}
$$

It is worth noting that the solution of this example possesses primary discontinuous points $t=0,1$, where

$$
0=y^{(k)}(0-) \neq y^{(k)}(0+) \quad \text { and } \quad y^{(k)}(1-) \neq y^{(k)}(1+), \quad k \geq 1 .
$$

Figure 2 plots the errors for $4 \leq N \leq 24$ in both $L^{\infty}$ and $L^{2}$ norms. The corresponding errors versus several values of $N$ are displayed in Table 2. The spectral accuracy is obtained although the solution for the equation is unsmooth at the primary discontinuous points.

\begin{tabular}{rcccccc}
\hline$N$ & 4 & 8 & 12 & 16 & 20 & 24 \\
\hline$L^{\infty}$-error for $e_{0}$ & $3.99 \cdot 10^{-01}$ & $1.12 \cdot 10^{-04}$ & $7.43 \cdot 10^{-09}$ & $7.39 \cdot 10^{-13}$ & $8.38 \cdot 10^{-13}$ & $1.89 \cdot 10^{-12}$ \\
$L^{2}$-error for $e_{0}$ & $2.86 \cdot 10^{-01}$ & $8.06 \cdot 10^{-05}$ & $5.37 \cdot 10^{-09}$ & $2.10 \cdot 10^{-13}$ & $1.42 \cdot 10^{-13}$ & $1.46 \cdot 10^{-13}$ \\
$L^{\infty}$-error for $e_{1}$ & $1.10 \cdot 10^{-00}$ & $3.42 \cdot 10^{-04}$ & $2.37 \cdot 10^{-08}$ & $1.53 \cdot 10^{-12}$ & $2.50 \cdot 10^{-12}$ & $5.06 \cdot 10^{-12}$ \\
$L^{2}$-error for $e_{1}$ & $7.65 \cdot 10^{-01}$ & $2.44 \cdot 10^{-04}$ & $1.69 \cdot 10^{-08}$ & $5.39 \cdot 10^{-13}$ & $2.86 \cdot 10^{-13}$ & $3.75 \cdot 10^{-13}$ \\
\hline
\end{tabular}

Table 2. Example 2: Errors versus $N$ in $L^{\infty}$ and $L^{2}$ norms. 
For the nonlinear VIDEs with nonvanishing delay of the form

$$
\begin{gathered}
y^{\prime}(t)=d(t, y(t), y(\theta(t)))+\int_{0}^{t} K_{1}(t, s, y(s)) d s+\int_{0}^{\theta(t)} K_{2}(t, s, y(s)) d s, \\
t \in(0, T], \\
y(t)=\phi(t), \quad t \in[\theta(0), 0],
\end{gathered}
$$

we can design a spectral-collocation method similar to the linear case. Equation (87) can be written as

$$
\begin{gathered}
u^{\prime}(x)=h(x, u(x), u(\vartheta(x)))+\int_{-1}^{x} R_{1}(x, z, u(z)) d z+\int_{-1}^{\vartheta(x)} R_{2}(x, z, u(z)) d z, \\
z \in(-1,1], \\
u(x)=\psi(x), \quad x \in[\vartheta(-1),-1],
\end{gathered}
$$

where

$$
\begin{gathered}
u(x):=y(t(x)), \quad \vartheta(x):=\frac{2}{T} \theta(t(x))-1, \quad \psi(x):=\phi(t(x)), \\
h(x, u(x), u(\vartheta(x)))=\frac{T}{2} d(t(x), y(t(x)), y(\theta(t(x)))), \\
R_{1}(x, z, u(z)):=\left(\frac{T}{2}\right)^{2} K_{1}(t(x), s(z), y(s(z))), \\
R_{2}(x, z, u(z)):=\left(\frac{T}{2}\right)^{2} K_{2}(t(x), s(z), y(s(z))) .
\end{gathered}
$$

We assume that (88) holds at the collocation points $x_{i}^{\mu}$, where $i=0,1, \ldots, n$ and $\mu=0,1, \ldots, M$ :

$$
\begin{aligned}
u^{\prime}\left(x_{i}^{\mu}\right)=h & \left(x_{i}^{\mu}, u\left(x_{i}^{\mu}\right), u\left(\vartheta\left(x_{i}^{\mu}\right)\right)\right)+\int_{-1}^{x_{i}^{\mu}} R_{1}\left(x_{i}^{\mu}, z, u(z)\right) d z \\
& +\int_{-1}^{\vartheta\left(x_{i}^{\mu}\right)} R_{2}\left(x_{i}^{\mu}, z, u(z)\right) d z \\
= & h\left(x_{i}^{\mu}, u\left(x_{i}^{\mu}\right), u\left(\vartheta\left(x_{i}^{\mu}\right)\right)\right)+\sum_{r=0}^{\mu-1} \int_{\eta_{r}}^{\eta_{r+1}} R_{1}\left(x_{i}^{\mu}, z, u(z)\right) d z \\
& +\int_{\eta_{\mu}}^{x_{i}^{\mu}} R_{1}\left(x_{i}^{\mu}, z, u(z)\right) d z \\
& +\sum_{r=0}^{\mu-2} \int_{\eta_{r}}^{\eta_{r+1}} R_{2}\left(x_{i}^{\mu}, z, u(z)\right) d z+\int_{\eta_{\mu-1}}^{\vartheta\left(x_{i}^{\mu}\right)} R_{2}\left(x_{i}, z\right) u(z) d z .
\end{aligned}
$$

We use $u_{i}^{\mu}$ to approximate $u\left(x_{i}^{\mu}\right), v_{i}^{\mu}$ to approximate $u\left(\vartheta\left(x_{i}^{\mu}\right)\right), \rho_{i}^{\mu}$ to approximate $u^{\prime}\left(x_{i}^{\mu}\right), i=0,1, \ldots, N, \mu=0,1, \ldots, M$, and use $u^{N}(x)$ to approximate $u(x)$, 
$\rho^{N}(x)$ to approximate $u^{\prime}(x)$. Similarly to (17), the numerical scheme for (88) is

$$
\rho_{i}^{\mu}=h\left(x_{i}^{\mu}, u_{i}^{\mu}, v_{i}^{\mu}\right)+\varpi\left(x_{i}^{\mu}\right)+\gamma\left(x_{i}^{\mu}\right),
$$

where

$$
\begin{aligned}
\varpi\left(x_{i}^{\mu}\right) & =\sum_{r=0}^{\mu-1} \frac{\eta_{r+1}-\eta_{r}}{2} \varpi_{1}^{r}\left(x_{i}^{\mu}\right)+\frac{\eta_{\mu+1}-\eta_{\mu}}{2} \frac{x_{i}+1}{2} \varpi_{3}\left(x_{i}^{\mu}\right), \quad \mu \geq 0, \\
\varpi_{1}^{r}\left(x_{i}^{\mu}\right) & :=\sum_{k=0}^{N} R_{1}\left(x_{i}^{\mu}, z_{r}\left(v_{k}\right), u_{k}^{r}\right) \omega_{k}, \quad r=0,1, \ldots, \mu-1, \\
\varpi_{3}\left(x_{i}^{\mu}\right) & :=\sum_{k=0}^{N} R_{1}\left(x_{i}^{\mu}, z_{\mu}\left(z\left(-1, x_{i}, v_{k}\right)\right), \sum_{j=0}^{N} u_{j}^{\mu} F_{j}\left(z\left(-1, x_{i}, v_{k}\right)\right)\right) \omega_{k},
\end{aligned}
$$

and

$$
\gamma\left(x_{i}^{\mu}\right)=\left\{\begin{array}{cl}
\frac{\vartheta\left(x_{i}^{0}\right)+1}{2} \gamma_{2}\left(x_{i}^{0}\right), & \mu=0, \\
\sum_{r=0}^{\mu-2} \frac{\eta_{r+1}-\eta_{r}}{2} \gamma_{1}^{r}\left(x_{i}^{\mu}\right)+\frac{\eta_{\mu}-\eta_{\mu-1}}{2} \frac{\widetilde{\vartheta}\left(x_{i}^{\mu}\right)+1}{2} \gamma_{3}\left(x_{i}^{\mu}\right), & \mu>0,
\end{array}\right.
$$$$
\gamma_{1}^{r}\left(x_{i}^{\mu}\right):=\sum_{k=0}^{N} R_{2}\left(x_{i}^{\mu}, z_{r}\left(v_{k}\right), u_{k}^{r}\right) \omega_{k}, \quad r=0,1, \ldots, \mu-2,
$$$$
\gamma_{2}\left(x_{i}^{0}\right):=\sum_{k=0}^{N} R_{2}\left(x_{i}^{0}, z\left(-1, \vartheta\left(x_{i}^{0}\right), v_{k}\right), \psi\left(z\left(-1, \vartheta\left(x_{i}^{0}\right), v_{k}\right)\right)\right) \omega_{k},
$$$$
\gamma_{3}\left(x_{i}^{\mu}\right):=\sum_{k=0}^{N} R_{2}\left(x_{i}^{\mu}, z_{\mu-1}\left(z\left(-1, \widetilde{\vartheta}\left(x_{i}^{\mu}\right), v_{k}\right)\right), \sum_{j=0}^{N} u_{j}^{\mu-1} F_{j}\left(z\left(-1, \widetilde{\vartheta}\left(x_{i}^{\mu}\right), v_{k}\right)\right)\right) \omega_{k} \text {. }
$$

Combining (19) with (22) and (91), we obtain the numerical scheme for the nonlinear VIDEs (87).

Example 3. Consider (87) with

$T=2, \quad \phi(t)=1, \quad \theta(t)=t-1, \quad d(t, y(t), y(\theta(t)))=y^{2}(\theta(t))-1-e^{2 t-1}+2 e^{t}$,

$$
K_{1}(t, s, y(s))=0, \quad K_{2}(t, s, y(s))=e^{t+s} y^{2}(s) .
$$

The corresponding exact solution is

$$
y(t)=\left\{\begin{array}{cc}
e^{t}, & t \in(0,1], \\
\frac{1}{2} e^{2 t-2}-\frac{1}{2} e^{2 t-1}+\frac{5}{3} e^{t}+\frac{1}{12} e^{4 t-3}-t+\frac{1}{4} e+\frac{1}{2}, & t \in(1,2] .
\end{array}\right.
$$

In this example, the primary discontinuous points are $t=0,1$, where

$$
0=y^{(k)}(0-) \neq y^{(k)}(0+) \quad \text { and } \quad y^{(k)}(1-) \neq y^{(k)}(1+), \quad k \geq 1 .
$$

Numerical errors versus several values of $N$ are displayed in Table 3 and Figure 3. These results indicate that the desired spectral accuracy is obtained. 


\begin{tabular}{rcccccc}
\hline$N$ & 4 & 8 & 12 & 16 & 20 & 24 \\
\hline$L^{\infty}$-error for $e_{0}$ & $5.67 \cdot 10^{-02}$ & $1.56 \cdot 10^{-05}$ & $8.55 \cdot 10^{-10}$ & $1.15 \cdot 10^{-13}$ & $1.28 \cdot 10^{-13}$ & $3.06 \cdot 10^{-13}$ \\
$L^{2}$-error for $e_{0}$ & $3.51 \cdot 10^{-02}$ & $9.75 \cdot 10^{-06}$ & $5.36 \cdot 10^{-10}$ & $3.00 \cdot 10^{-14}$ & $2.23 \cdot 10^{-14}$ & $2.24 \cdot 10^{-14}$ \\
$L^{\infty}$-error for $e_{1}$ & $2.12 \cdot 10^{-01}$ & $6.13 \cdot 10^{-05}$ & $3.40 \cdot 10^{-09}$ & $3.55 \cdot 10^{-13}$ & $3.48 \cdot 10^{-13}$ & $9.02 \cdot 10^{-13}$ \\
$L^{2}$-error for $e_{1}$ & $1.28 \cdot 10^{-01}$ & $3.80 \cdot 10^{-05}$ & $2.11 \cdot 10^{-09}$ & $8.71 \cdot 10^{-14}$ & $6.06 \cdot 10^{-14}$ & $6.39 \cdot 10^{-14}$ \\
\hline
\end{tabular}

Table 3. Example 3: Errors versus $N$ in $L^{\infty}$ and $L^{2}$ norms.

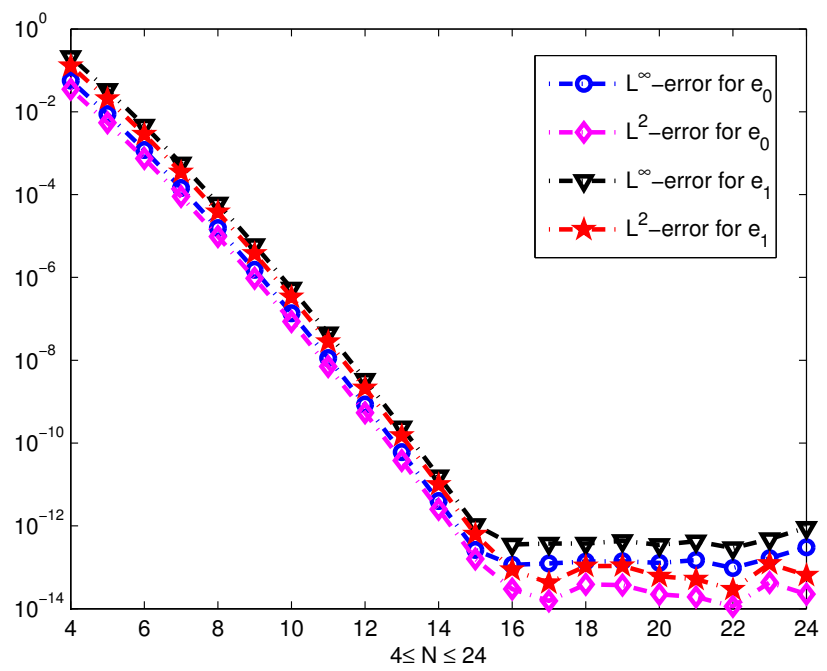

Figure 3. Example 3: Errors versus $N$ in $L^{\infty}$ and $L^{2}$ norms.

Example 4. Now we consider the case where the delay is a function of the solution; i.e.,

$$
\begin{gathered}
y^{\prime}(t)=d(t, y(t), y(\theta(t, y(t))))+\int_{0}^{t} K_{1}(t, s, y(s)) d s+\int_{0}^{\theta(t, y(t))} K_{2}(t, s, y(s)) d s, \\
t \in[0, T], \\
y(t)=\phi(t), \quad t \in[\theta(0, y(0)), 0] .
\end{gathered}
$$

If we take

$$
\begin{gathered}
T=2, \quad \phi(t)=2.5, \quad \theta(t, y(t))=t-y(t), \\
d(t, y(t), y(\theta(t, y(t))))=y^{2}(t)+y^{2}(\theta(t, y(t)))+g(t), \\
g(t)=-\frac{1}{2} e^{-t}-\frac{1}{4}\left(e^{-t}+4\right)^{2} \\
-\frac{1}{4} e^{t}\left(-\frac{1}{3} e^{-3 t}-4 e^{-2 t}-16 e^{-t}+\frac{1}{3}+20\right)-(2.5)^{2}\left(t-\frac{1}{2} e^{-t}-1\right), \\
K_{1}(t, s, y(s))=e^{t-s} y^{2}(s), \quad K_{2}(t, s, y(s))=y^{2}(s),
\end{gathered}
$$




\begin{tabular}{rcccccc}
\hline$N$ & 2 & 4 & 6 & 8 & 10 & 12 \\
\hline$L^{\infty}$-error for $e_{0}$ & $3.32 \cdot 10^{-02}$ & $5.33 \cdot 10^{-04}$ & $8.78 \cdot 10^{-07}$ & $2.87 \cdot 10^{-09}$ & $6.54 \cdot 10^{-12}$ & $2.22 \cdot 10^{-14}$ \\
$L^{2}$-error for $e_{0}$ & $3.05 \cdot 10^{-02}$ & $2.50 \cdot 10^{-04}$ & $7.65 \cdot 10^{-07}$ & $2.62 \cdot 10^{-09}$ & $5.91 \cdot 10^{-12}$ & $1.24 \cdot 10^{-14}$ \\
$L^{\infty}$-error for $e_{1}$ & $1.57 \cdot 10^{-01}$ & $1.14 \cdot 10^{-03}$ & $1.56 \cdot 10^{-06}$ & $3.99 \cdot 10^{-09}$ & $8.29 \cdot 10^{-12}$ & $3.42 \cdot 10^{-14}$ \\
$L^{2}$-error for $e_{1}$ & $8.73 \cdot 10^{-02}$ & $6.41 \cdot 10^{-04}$ & $1.06 \cdot 10^{-06}$ & $3.16 \cdot 10^{-09}$ & $6.75 \cdot 10^{-12}$ & $1.66 \cdot 10^{-14}$ \\
\hline
\end{tabular}

Table 4. Example 4: Errors versus $N$ in $L^{\infty}$ and $L^{2}$ norms.

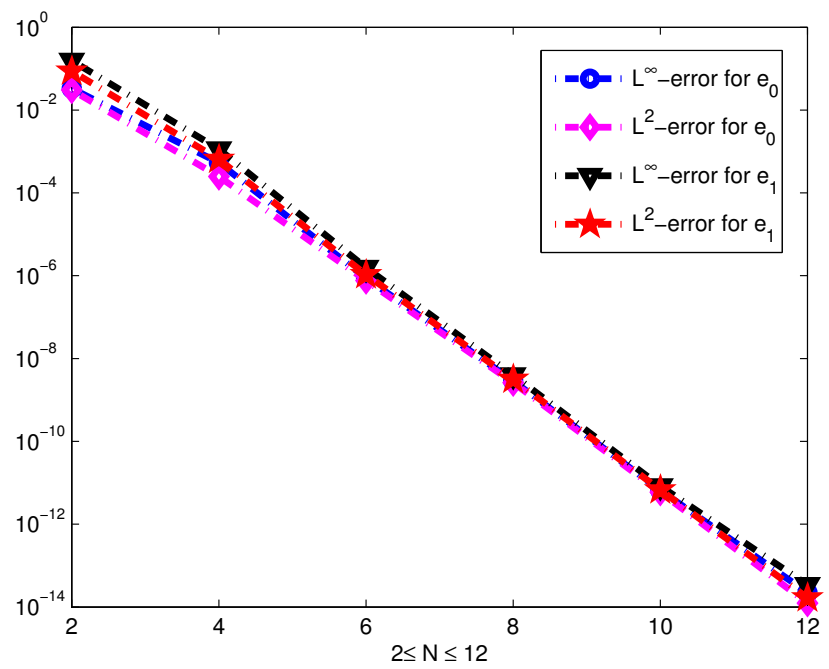

Figure 4. Example 4: Errors versus $N$ in $L^{\infty}$ and $L^{2}$ norms.

then the corresponding exact solution is

$$
y(t)=\left\{\begin{array}{cl}
2.5, & t \in[-1,0], \\
\frac{1}{2}\left(e^{-t}+4\right), & t \in(0,2] .
\end{array}\right.
$$

This solution possesses a primary discontinuous point $t=0$ where $y^{(k)}(0-)=0$ while $y^{(k)}(0+)=(-1)^{k} \frac{1}{2}, k \geq 1$.

We use the Newton iterative method to solve the nonlinear discrete system corresponding to this example. Errors versus $N$ are listed in Table 4 and plotted in Figure 4 from which we can see that the spectral accuracy is obtained. This example shows that our method can also handle the case where the delay is a function of the solution.

\section{Conclusion and future work}

We propose the Legendre spectral-collocation method to solve VIDEs with nonvanishing delay, and provide convergence analysis for the proposed method. Numerical 
examples are provided to confirm the theoretical results that the numerical errors decay exponentially. The main difficulty in applying the spectral method to VIDEs with nonvanishing delay is the solution of this equation possesses primary discontinuous points associated with the nonvanishing delay function. We overcome this difficulty by dividing the global definition domain of the solution into several subintervals where the solution is smooth enough. Then spectral method can be used to approximate the solution in each subinterval.

Our future work will focus on the spectral method for the Volterra functional integral and differential integral equation with nonvanishing delay.

\section{References}

[1] K. Al-Khaled, Numerical approximations for population growth models, Appl. Math. Comput. 160 (2005), no. 3, 865-873. MR 2005i:65113 Zbl 1062.65142

[2] I. Ali, Convergence analysis of spectral methods for integro-differential equations with vanishing proportional delays, J. Comput. Math. 29 (2011), no. 1, 49-60. MR 2012b:65214 Zbl 1249.65281

[3] I. Ali, H. Brunner, and T. Tang, A spectral method for pantograph-type delay differential equations and its convergence analysis, J. Comput. Math. 27 (2009), no. 2-3, 254-265. MR 2010f: 65197 Zbl 1212.65308

[4] I. Ali, H. Brunner, and T. Tang, Spectral methods for pantograph-type differential and integral equations with multiple delays, Front. Math. China 4 (2009), no. 1, 49-61. MR 2009m:65099 Zbl 05567165

[5] C. T. H. Baker and N. J. Ford, Asymptotic error expansions for linear multistep methods for a class of delay integro-differential equations, Bull. Soc. Math. Grèce (N.S.) 31 (1990), 5-18. MR 92e:65170 Zbl 0746.65097

[6] C. T. H. Baker and C. A. H. Paul, Parallel continuous Runge-Kutta methods and vanishing lag delay differential equations, Adv. Comput. Math. 1 (1993), no. 3-4, 367-394. MR 94g:65140 Zbl 0824.65055

[7] C. T. H. Baker and A. Tang, Stability analysis of continuous implicit Runge-Kutta methods for Volterra integro-differential systems with unbounded delays, Appl. Numer. Math. 24 (1997), no. 2-3, 153-173. MR 99a:45021 Zbl 0878.65122

[8] A. Bellen and M. Zennaro, Numerical methods for delay differential equations, Clarendon/Oxford University Press, New York, 2003. MR 2004i:65001 Zbl 1038.65058

[9] G. A. Bocharov and F. A. Rihan, Numerical modelling in biosciences using delay differential equations, J. Comput. Appl. Math. 125 (2000), no. 1-2, 183-199. MR 1803191 Zbl 0969.65124

[10] F. Brauer and P. van den Driessche, Models for transmission of disease with immigration of infectives, Math. Biosci. 171 (2001), no. 2, 143-154. MR 2002b:92035 Zbl 0995.92041

[11] H. Brunner, Collocation methods for nonlinear Volterra integro-differential equations with infinite delay, Math. Comp. 53 (1989), no. 188, 571-587. MR 90m:65227 Zbl 0681.65105

[12] _ The numerical solution of neutral Volterra integro-differential equations with delay arguments, Ann. Numer. Math. 1 (1994), no. 1-4, 309-322. MR 96m:45014 Zbl 0828.65146

[13] __ Collocation methods for Volterra integral and related functional differential equations, Cambridge Monographs on Applied and Computational Mathematics, no. 15, Cambridge University Press, 2004. MR 2005k:65002 Zbl 1059.65122 
[14] _ Recent advances in the numerical analysis of Volterra functional differential equations with variable delays, J. Comput. Appl. Math. 228 (2009), no. 2, 524-537. MR 2010c:65251 Zbl 1170.65103

[15] C. Canuto, M. Y. Hussaini, A. Quarteroni, and T. A. Zang, Spectral methods: fundamentals in single domains, Springer, Berlin, 2006. MR 2007c:65001 Zbl 1093.76002

[16] J. Chattopadhyay, R. R. Sarkar, and A. el Abdllaoui, A delay differential equation model on harmful algal blooms in the presence of toxic substances, Math. Med. Biol. 19 (2002), no. 2, 137-161. Zbl 1013.92046

[17] Y. Chen, X. Li, and T. Tang, A note on Jacobi spectral-collocation methods for weakly singular Volterra integral equations with smooth solutions, J. Comput. Math. 31 (2013), no. 1, 47-56. MR 3021419

[18] Y. Chen and T. Tang, Spectral methods for weakly singular Volterra integral equations with smooth solutions, J. Comput. Appl. Math. 233 (2009), no. 4, 938-950. MR 2010k:65304 Zbl 1186.65161

[19] _ Convergence analysis of the Jacobi spectral-collocation methods for Volterra integral equations with a weakly singular kernel, Math. Comp. 79 (2010), no. 269, 147-167. MR 2011c:65298 Zbl 1207.65157

[20] J. M. Cushing, Integrodifferential equations and delay models in population dynamics, Lecture Notes in Biomathematics, no. 20, Springer, Berlin, 1977. MR 58 \#15300 Zbl 0363.92014

[21] W. H. Enright and M. Hu, Continuous Runge-Kutta methods for neutral Volterra integrodifferential equations with delay, Appl. Numer. Math. 24 (1997), no. 2-3, 175-190. MR 98g: $65126 \mathrm{Zbl} 0876.65089$

[22] D. J. Evans and K. R. Raslan, The Adomian decomposition method for solving delay differential equation, Int. J. Comput. Math. 82 (2005), no. 1, 49-54. MR 2159285 Zbl 1069.65074

[23] S. A. Gourley, Y. Kuang, and J. D. Nagy, Dynamics of a delay differential equation model of hepatitis B virus infection, J. Biol. Dyn. 2 (2008), no. 2, 140-153. MR 2009g:92047 Zbl 1140.92014

[24] J. S. Hesthaven, From electrostatics to almost optimal nodal sets for polynomial interpolation in a simplex, SIAM J. Numer. Anal. 35 (1998), no. 2, 655-676. MR 99b:65009 Zbl 0933.41004

[25] J. S. Hesthaven and T. Warburton, Nodal discontinuous Galerkin methods: algorithms, analysis, and applications, Texts in Applied Mathematics, no. 54, Springer, New York, 2008. MR 2008k:65002 Zbl 1134.65068

[26] D. Jiang, J. Wei, and B. Zhang, Positive periodic solutions of functional differential equations and population models, Electron. J. Differential Equations 2002 (2002), Article ID \#71. MR 2003i:34161 Zbl 1010.34065

[27] Y.-J. Jiang, On spectral methods for Volterra-type integro-differential equations, J. Comput. Appl. Math. 230 (2009), no. 2, 333-340. MR 2010d:45011 Zbl 1202.65170

[28] Y. Kuang, Delay differential equations with applications in population dynamics, Mathematics in Science and Engineering, no. 191, Academic Press, Boston, 1993. MR 94f:34001 Zbl 0777.34002

[29] S. Li, High order contractive Runge-Kutta methods for Volterra functional differential equations, SIAM J. Numer. Anal. 47 (2010), no. 6, 4290-4325. MR 2011a:65167 Zbl 1221.65166

[30] X. Li and T. Tang, Convergence analysis of Jacobi spectral collocation methods for AbelVolterra integral equations of second kind, Front. Math. China 7 (2012), no. 1, 69-84. MR 2876899 Zbl 1260.65111 
[31] T. Lin, Y. Lin, M. Rao, and S. Zhang, Petrov-Galerkin methods for linear Volterra integrodifferential equations, SIAM J. Numer. Anal. 38 (2000), no. 3, 937-963. MR 2001e:65227 Zbl 0983.65138

[32] A. Makroglou, A block-by-block method for the numerical solution of Volterra delay integrodifferential equations, Computing 30 (1983), no. 1, 49-62. MR 85f:65125 Zbl 0499.65064

[33] P. Nevai, Mean convergence of Lagrange interpolation, III, Trans. Amer. Math. Soc. 282 (1984), no. 2, 669-698. MR 85c:41009 Zbl 0577.41001

[34] H. J. Oberle and H. J. Pesch, Numerical treatment of delay differential equations by Hermite interpolation, Numer. Math. 37 (1981), no. 2, 235-255. MR 83a:65077 Zbl 0469.65057

[35] J. M. Ortega, Numerical analysis: a second course, Academic Press, New York, 1972. MR 53 \#6967 Zbl 0248.65001

[36] B. V. Riley, The numerical solution of Volterra integral equations with nonsmooth solutions based on sinc approximation, Appl. Numer. Math. 9 (1992), no. 3-5, 249-257. MR 93b:65210 Zbl 0757.65148

[37] M. Shakourifar and M. Dehghan, On the numerical solution of nonlinear systems of Volterra integro-differential equations with delay arguments, Computing 82 (2008), no. 4, 241-260. MR 2009j:45019 Zbl 1154.65098

[38] M. Shakourifar and W. H. Enright, Reliable approximate solution of systems of Volterra integrodifferential equations with time-dependent delays, SIAM J. Sci. Comput. 33 (2011), no. 3, 1134-1158. MR 2012e:65325 Zbl 1233.65101

[39] _ Superconvergent interpolants for collocation methods applied to Volterra integrodifferential equations with delay, BIT 52 (2012), no. 3, 725-740. MR 2965299 Zbl 1255.65250

[40] L. F. Shampine and S. Thompson, Solving DDEs in MATLAB, Appl. Numer. Math. 37 (2001), no. 4, 441-458. MR 2002c:65106 Zbl 0983.65079

[41] J. Shen and T. Tang, Spectral and high-order methods with applications, Mathematics Monograph Series, no. 3, Science Press, Beijing, 2006. MR 2012b:65001 Zbl 1234.65005

[42] T. Tang and X. Xu, Accuracy enhancement using spectral postprocessing for differential equations and integral equations, Commun. Comput. Phys. 5 (2009), no. 2-4, 779-792. MR 2010d: 65178

[43] T. Tang, X. Xu, and J. Cheng, On spectral methods for Volterra integral equations and the convergence analysis, J. Comput. Math. 26 (2008), no. 6, 825-837. MR 2010c:65256 Zbl 1174.65058

[44] S. Thompson and L. F. Shampine, A friendly Fortran DDE solver, Appl. Numer. Math. 56 (2006), no. 3-4, 503-516. MR 2207606 Zbl 1089.65062

[45] M. Villasana and A. Radunskaya, A delay differential equation model for tumor growth, J. Math. Biol. 47 (2003), no. 3, 270-294. MR 2004j:92012 Zbl 1023.92014

[46] V. Volterra, Variazioni e fluttuazioni del numero d'individui in specie animali conviventi, Memorie del Regio Comitato Talassografico Italiano 131 (1927). JFM 52.0450.06

[47] _ The general equations of biological strife in the case of historical actions, Proc. Edinburgh Math. Soc. (2) 6 (1939), no. 1, 4-10. Zbl 0021.34003

[48] Z. Wan, Y. Chen, and Y. Huang, Legendre spectral Galerkin method for second-kind Volterra integral equations, Front. Math. China 4 (2009), no. 1, 181-193. MR 2009k:65285 Zbl 05567172

[49] Y. Wei and Y. Chen, Convergence analysis of the Legendre spectral collocation methods for second order Volterra integro-differential equations, Numer. Math. Theory Methods Appl. 4 (2011), no. 3, 419-438. MR 2012k:65177 Zbl 1265.65278 
[50] _ Convergence analysis of the spectral methods for weakly singular Volterra integrodifferential equations with smooth solutions, Adv. Appl. Math. Mech. 4 (2012), no. 1, 1-20. MR 2876648 Zbl 1262.45005

[51] __ Legendre spectral collocation methods for pantograph Volterra delay-integro-differential equations, J. Sci. Comput. 53 (2012), no. 3, 672-688. MR 2996451 Zbl 1264.65218

[52] Z. Xie, X. Li, and T. Tang, Convergence analysis of spectral Galerkin methods for Volterra type integral equations, J. Sci. Comput. 53 (2012), no. 2, 414-434. MR 2983100 Zbl 06198196

[53] C. Zhang and S. Vandewalle, General linear methods for Volterra integro-differential equations with memory, SIAM J. Sci. Comput. 27 (2006), no. 6, 2010-2031. MR 2006k:65394 Zbl 1104.65133

[54] W. Zhang and M. Fan, Periodicity in a generalized ecological competition system governed by impulsive differential equations with delays, Math. Comput. Modelling 39 (2004), no. 4-5, 479-493. MR 2046535 Zbl 1065.92066

[55] H. ZivariPiran and W. H. Enright, An efficient unified approach for the numerical solution of delay differential equations, Numer. Algorithms 53 (2010), no. 2-3, 397-417. MR 2011j:65133 Zbl 1184.65071

Received November 17, 2012. Revised August 28, 2013.

YANPING CHEN: yanpingchen@scnu.edu.cn

School of Mathematics Science, South China Normal University, Guangzhou 510631, China

ZHENDONG GU: guzhd@qq.com

School of Mathematics and Computational Science, Xiangtan University, Xiangtan 411105, Hunan, China 


\title{
Communications in Applied Mathematics and Computational Science
}

\author{
msp.org/camcos
}

EDITORS

MANAGING EDITOR

John B. Bell

Lawrence Berkeley National Laboratory, USA

jbbell@lbl.gov

\section{BOARD OF EDITORS}

\begin{tabular}{|c|c|c|c|}
\hline Marsha Berger & $\begin{array}{l}\text { New York University } \\
\text { berger@cs.nyu.edu }\end{array}$ & Ahmed Ghoniem & $\begin{array}{l}\text { Massachusetts Inst. of Technology, USA } \\
\text { ghoniem@mit.edu }\end{array}$ \\
\hline Alexandre Chorin & $\begin{array}{l}\text { University of California, Berkeley, USA } \\
\text { chorin@math.berkeley.edu }\end{array}$ & Raz Kupferman & $\begin{array}{l}\text { The Hebrew University, Israel } \\
\text { raz@math.huji.ac.il }\end{array}$ \\
\hline Phil Colella & $\begin{array}{l}\text { Lawrence Berkeley Nat. Lab., USA } \\
\text { pcolella@lbl.gov }\end{array}$ & Randall J. LeVeque & $\begin{array}{l}\text { University of Washington, USA } \\
\text { rj1@ amath.washington.edu }\end{array}$ \\
\hline Peter Constantin & $\begin{array}{l}\text { University of Chicago, USA } \\
\text { const@cs.uchicago.edu }\end{array}$ & Mitchell Luskin & $\begin{array}{l}\text { University of Minnesota, USA } \\
\text { luskin@umn.edu }\end{array}$ \\
\hline Maksymilian Dryja & $\begin{array}{l}\text { Warsaw University, Poland } \\
\text { maksymilian.dryja@acn.waw.pl }\end{array}$ & Yvon Maday & $\begin{array}{l}\text { Université Pierre et Marie Curie, France } \\
\text { maday@ann.jussieu.fr }\end{array}$ \\
\hline M. Gregory Forest & $\begin{array}{l}\text { University of North Carolina, USA } \\
\text { forest@amath.unc.edu }\end{array}$ & James Sethian & $\begin{array}{l}\text { University of California, Berkeley, USA } \\
\text { sethian@ math.berkeley.edu }\end{array}$ \\
\hline Leslie Greengard & $\begin{array}{l}\text { New York University, USA } \\
\text { greengard@ cims.nyu.edu }\end{array}$ & Juan Luis Vázquez & $\begin{array}{l}\text { Universidad Autónoma de Madrid, Spain } \\
\text { juanluis.vazquez@uam.es }\end{array}$ \\
\hline Rupert Klein & $\begin{array}{l}\text { Freie Universität Berlin, Germany } \\
\text { rupert.klein@pik-potsdam.de }\end{array}$ & Alfio Quarteroni & $\begin{array}{l}\text { Ecole Polytech. Féd. Lausanne, Switzerland } \\
\text { alfio.quarteroni@epfl.ch }\end{array}$ \\
\hline \multirow[t]{2}{*}{ Nigel Goldenfeld } & $\begin{array}{l}\text { University of Illinois, USA } \\
\text { nigel@uiuc.edu }\end{array}$ & Eitan Tadmor & $\begin{array}{l}\text { University of Maryland, USA } \\
\text { etadmor@cscamm.umd.edu }\end{array}$ \\
\hline & & Denis Talay & $\begin{array}{l}\text { INRIA, France } \\
\text { denis.talay@inria.fr }\end{array}$ \\
\hline
\end{tabular}

\section{PRODUCTION}

production@msp.org

Silvio Levy, Scientific Editor

See inside back cover or msp.org/camcos for submission instructions.

The subscription price for 2013 is US \$75/year for the electronic version, and \$105/year ( $\$ 15$, if shipping outside the US) for print and electronic. Subscriptions, requests for back issues from the last three years and changes of subscribers address should be sent to MSP.

Communications in Applied Mathematics and Computational Science (ISSN 2157-5452 electronic, 1559-3940 printed) at Mathematical Sciences Publishers, 798 Evans Hall \#3840, c/o University of California, Berkeley, CA 94720-3840, is published continuously online. Periodical rate postage paid at Berkeley, CA 94704, and additional mailing offices.

CAMCoS peer review and production are managed by EditFLOW ${ }^{\circledR}$ from Mathematical Sciences Publishers.

\section{PUBLISHED BY}

mathematical sciences publishers

nonprofit scientific publishing

http://msp.org/

(C) 2013 Mathematical Sciences Publishers 


\section{Communications in Applied Mathematics and Computational Science}

vol. 8

no. 1

2013

On the origin of divergence errors in MHD simulations and consequences for $\quad I$ numerical schemes

FRIEDEMANN KEMM

Renormalized reduced models for singular PDEs

PANOS StINIS

Legendre spectral-collocation method for Volterra integral differential equations with nonvanishing delay

YANPING Chen and ZHendong GU

A cartesian grid embedded boundary method for the compressible Navier-Stokes equations

Daniel T. Graves, Phillip Colella, David Modiano, Jeffrey Johnson, Bjorn Sjogreen and Xinfeng GaO

Second-order accuracy of volume-of-fluid interface reconstruction algorithms

II: An improved constraint on the cell size

\section{Elbridge Gerry Puckett}

Computational models of material interfaces for the study of extracorporeal shock wave therapy

Kirsten Fagnan, Randall J. LeVeque and Thomas J. Matula 